\title{
Nonlinear Vibrations of FGM Cylindrical Panel with Simply Supported Edges in Air Flow
}

\author{
Y. X. Hao, ${ }^{1}$ W. Zhang, ${ }^{2}$ S. B. Li, ${ }^{3}$ and J. H. Zhang ${ }^{1}$ \\ ${ }^{1}$ College of Mechanical Engineering, Beijing Information Science and Technology University, Beijing 100192, China \\ ${ }^{2}$ College of Mechanical Engineering, Beijing University of Technology, Beijing 100124, China \\ ${ }^{3}$ College of Science, Civil Aviation University of China, Tianjin 300300, China
}

Correspondence should be addressed to W. Zhang; sandyzhang0@yahoo.com

Received 6 June 2014; Accepted 9 December 2014

Academic Editor: Mahmut Reyhanoglu

Copyright (C) 2015 Y. X. Hao et al. This is an open access article distributed under the Creative Commons Attribution License, which permits unrestricted use, distribution, and reproduction in any medium, provided the original work is properly cited.

\begin{abstract}
Chaotic and periodic motions of an FGM cylindrical panel in hypersonic flow are investigated. The cylindrical panel is also subjected to in-plane external loads and a linear temperature variation in the thickness direction. The temperature dependent material properties of panel which are assumed to be changed through the thickness direction only can be determined by a simple power distribution in terms of the volume fractions. With Hamilton's principle for an elastic body, a nonlinear dynamical model based on Reddy's first-order shear deformation shell theory and von Karman type geometric nonlinear relationship is derived in the form of partial equations. A third-order piston theory is adopted to evaluate the hypersonic aerodynamic load. Here, Galerkin's method is employed to discretize this continuous nonlinear dynamic system to ordinary differential governing equations involving two degrees of freedom. The chaotic and periodic response are studied by the direct numerical simulation method for influences of different Mach number and the value of in-plane load. The bifurcations, Poincare section, waveform, and phase plots are presented.
\end{abstract}

\section{Introduction}

With the continuous variation of the material properties along the thickness, functionally graded materials (FGM) can be used in high temperature gradient environments especially when they are made of metal and ceramic. The metal can keep a certain extent of toughness and ceramics have superior heat resistant ability. So they usually act as thermal protection structures in spacecraft and other structural components in high temperature environments $[1,2]$. It is well known that, due to the combined load of airflow and heating, the flexible panels might exhibit large aerothermal deflections [3]. In an extensive search of panel flutter literature, a number of investigations were dedicated to FGM plates with supersonic or hypersonic flow regimes. Considered a curved skin panel with geometrical imperfection, Abbas et al. [4] gave its flutter in unsteady flow by numerical simulation and Galerkin method. With the help of structural nonlinear and the thirdorder piston theory, the governing equations were derived. The effects of the system parameters on the flutter were discussed in detail. Using the linear approach, the flutter of rectangular flat plates in supersonic flow and thermal environment was studied by Prakash and Ganapathi [5]. The plate was subjected to the two-dimensional aerodynamic force. They showed that under real flight conditions heating caused by aerodynamics is enormous. Sohn and Kim [6] took a static and dynamic stability study on the panel under aerodynamic force as well as thermal loads. Ibrahim et al. $[7,8]$ investigated the thermal buckling and nonlinear flutter of thin FGM panels under the action of aerothermoelasticity by the finite element method. Then they presented the results for different factors. Accounting for both the geometric and aerodynamic nonlinearities, Prakash et al. [9] studied the nonlinear flutter of FGM plates under high supersonic airflow in frequency domain and time domain, respectively. The influence of various parameters including the geometrics and physics on the flutter of FGM plates was discussed. Hosseini and Fazelzadeh [10] used the numerical and analytical methodologies to study postcritical and vibration behaviors of the FGM panels in a supersonic air flow. Ibrahim et al. [8] analyzed nonlinear flutter and thermal buckling of an FGM 
panel under the combined effect of elevated temperature conditions and aerodynamic loading. Lee and Kim [11] dealt with the aerothermopostbuckling behaviors of the FGM panel in supersonic air flow. Limit-cycle vibration was found in this study by Newmark time integration method and Guyan reduction technique. Mey et al. [12] found that, in many studies which are about the flutter characteristics of FGM plates, the linear or nonlinear theory in conjunction with the first-order piston theory used to approximate the aerodynamic pressure was considered. The first-order piston theory is valid for sufficiently high supersonic Mach numbers $\left(\sqrt{2}<M_{\infty}<5\right)$ on surfaces with small geometric characteristics.

Since the first report of flutter instability for circular cylindrical shells, the studies of the aeroelastic stability of cylindrical shells in axial flow received extensive attention [13]. Numerous studies on the cylindrical shells focused on their flutter. Marzocca et al. [14] reviewed the corresponding researches that analyse the dynamic behavior of curved and flat panels exposed to supersonic flow fields. Hosseini et al. [15] analyzed the nonlinear response of FGM curved panels in high temperature supersonic air flows. The effects of curved panel height-rise and volume fraction index on the nonlinear dynamical behavior of the panel which is subjected to aerothermoelastic loads are investigated.

Librescu et al. [16] presented a theoretical investigation of the flutter and postflutter of the long thin-walled circular cylindrical panels in supersonic/hypersonic flow field. For the problems of the flutter boundaries about the simply supported functionally graded truncated conical shell subjected to supersonic air flow, Mahmoudkhani et al. [17] made aerothermoelastic analysis to predict the flutter boundaries. The flutter boundaries were obtained for the FGM conical shells with different semivertex cone angles, different temperature distributions, and different volume fraction indices.

When it comes to FGM cylindrical panel identification, damage detection, and the control of the dynamics, it is necessary to investigate their complex nonlinear flutter in hypersonic air flow in great detail [18]. However, to the best of the authors' knowledge, works on dynamic instability of FGM cylindrical panel subjected to supersonic/hypersonic flow, including the effects of thermal load and in-plane loads, appear to be scarce in the open literature. Many interesting researches used the linear shell theory. There are a few literatures on the nonlinear dynamic behavior of FGM cylindrical panel taking into account the aerodynamic nonlinearities.

In the present research, the bifurcations and chaotic dynamics of the hypersonic FGM cylindrical panel subjected to thermal and mechanical loads are investigated by applying geometrical nonlinear and the third-order piston theory. Materials properties of the constituents are graded in the thickness direction according to a power law distribution. Only transverse nonlinear oscillations of the FGM cylindrical panel are considered; the equations of motion can be reduced into a two-degree-of-freedom nonlinear system. By the numerical method, the nonlinear dynamical equations are analyzed to find the nonlinear responses of the system.

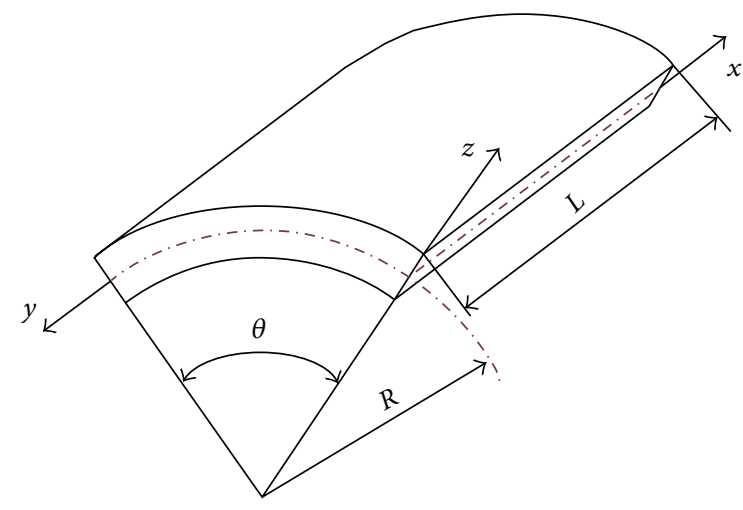

FIgURE 1: The model of an FGM cylindrical panel with simply supported edges and the coordinate system.

\section{Theoretical Formulation}

2.1. Model of the FGM Cylindrical Panel. Consider a simply supported hypersonic FGM circular cylindrical panel of a length $L$, thickness $h$, midsurface radius $R$, and angular width $\theta$. This panel is also subjected to the in-plane harmonic excitations. Cartesian coordinate $O x y z$ is adopted to describe the deformations of the FGM circular cylindrical panel. The coordinates $x$ and $y$ are in longitudinal and tangential directions, respectively. The coordinate $z$ is taken to be positive outward radially, as shown in Figure 1. The displacements of an arbitrary point are denoted by $u(x, y, z, t), v(x, y, z, t)$, and $w(x, y, z, t)$. Assume that $u_{0}(x, y, z, t), v_{0}(x, y, z, t)$, and $w_{0}(x, y, z, t)$ are the displacements of a point in the middle plane in the axial, circumferential, and radial directions, respectively. The rotations of the transverse normal to the midplane about $y$ and $x$ axes are assumed to be $\phi_{x}$ and $\phi_{y}$, respectively. The in-plane excitation of the FGM panel distributed uniformly along the $x$ direction at the end of panels $x=0$ and $x=L$ is of the form $p^{\prime}=-\left(p_{0}-p_{1}(t)\right)$, where $p_{0}$ is the static in-plane preload and $p_{1}(t)$ is part of time dependent that has the form $p_{1}(t)=-p_{1} \cos \left(\omega_{1} t\right)$. Here, $\omega_{1}$ is the frequency of excitation along axial direction.

Assume that the panel material is made of a composite of the ceramics and metals. The material properties of constituents of the panel including density $\rho$, elastic moduli $E$, and thermal coefficient of expansion $\alpha$ are temperature dependent and can be expressed as [19]

$$
\widetilde{P}=P_{0}\left(P_{-1} T^{-1}+1+P_{1} T+P_{2} T^{2}+P_{3} T^{3}\right),
$$

where $P_{0}, P_{-1}, P_{1}, P_{2}$, and $P_{3}$ are temperature dependent coefficients and $T$ is the environment temperature.

The effective material properties $P$ vary continuously in the $z$ direction by following a simple power law in terms of the volume fractions and can be expressed as

$$
P=P_{c} V_{c}+P_{m} V_{m}
$$

where $P_{c}$ and $P_{m}$ indicate, respectively, the properties of the ceramic and metal and $V_{c}$ and $V_{m}$ are their volume fractions and have the following relationship:

$$
V_{c}+V_{m}=1 ;
$$


the metal volume fraction $V_{m}$ can be written as

$$
V_{m}(z)=\left(\frac{z}{h}+\frac{1}{2}\right)^{\eta}, \quad \eta \geq 0
$$

where $\eta$ is the volume fraction exponent which can depict the material variation profile through the thickness. According to Zhang et al. [20], linear temperature change is considered which has the form

$$
T(z)=\frac{T_{t}+T_{b}}{2}+\frac{T_{t}-T_{b}}{h} z,
$$

where $T_{t}$ and $T_{b}$ indicate the temperature of the top and bottom surfaces of the panel, respectively. The FGM circular cylindrical panel is subjected to a uniform temperature variation $\Delta T=T-T_{0}$, where $T_{b}$ is the reference temperature.

2.2. Aerodynamics Loading. The linear piston theory is valid for Mach numbers changing from $\sqrt{2}$ to 5 and for higher Mach numbers the nonlinear piston theory must be used in Mei et al. [21]. To study the nonlinear oscillation of functionally graded material cylindrical panel under a hypersonic air flow, one should use third-order piston theory. Piston theory aerodynamics, which is used in problems of oscillating airfoils advanced by Lighthill [22] and later used as an aeroelastic tool by Ashley and Zartarian [23], is a popular modeling technique for supersonic and hypersonic aeroelastic analyses.

For a cylindrical panel, which is exposed to an external hypersonic flow field parallel to the centerline of the panel on the surface, the aerodynamic pressure $\Delta P$ using the thirdorder piston theory is expressed by Amabili [13] and Cao and Zhao [24] as

$$
\begin{aligned}
\Delta P=-\frac{2 p_{\infty}}{M_{a}}[ & \left(\frac{\partial w}{\partial x}+\frac{1}{U_{\infty}} \frac{\partial w}{\partial t}\right) \\
& +\frac{(1+\gamma) M_{a}}{4}\left(\frac{\partial w}{\partial x}+\frac{1}{U_{\infty}} \frac{\partial w}{\partial t}\right)^{2} \\
& \left.+\frac{(1+\gamma) M_{a}^{2}}{12}\left(\frac{\partial w}{\partial x}+\frac{1}{U_{\infty}} \frac{\partial w}{\partial t}\right)^{3}\right],
\end{aligned}
$$

where $\gamma$ is the adiabatic exponent and $M_{a}$ and $t$ are the Mach number and time, respectively. The free-stream static pressure is given as

$$
P_{\infty}=\frac{1}{2} \rho_{\infty} U_{\infty}^{2}, \quad U_{\infty}=M_{a} a_{\infty}
$$

where $\rho_{\infty}, U_{\infty}$, and $a_{\infty}$ are the free-stream air density, velocity, and the free-stream speed of sound, respectively.

2.3. Geometry and Constitutive Relations. The displacement components for the FGM cylindrical panel based on Reddy's first-order shear deformation theory [25] can be represented as

$$
\begin{gathered}
u(x, y, z, t)=u_{0}(x, y, t)+z \phi_{x}(x, y, t), \\
v(x, y, z, t)=v_{0}(x, y, t)+z \phi_{y}(x, y, t), \\
w(x, y, z, t)=w_{0}(x, y, t) .
\end{gathered}
$$

It is assumed that the transverse normal stress is negligible and normals are not vertical to the midplane after deformation. Substituting displacement components into Von Karman nonlinear strains-displacement relations, the strains in terms of middle-surface displacements are given as

$$
\begin{gathered}
\varepsilon_{x x}=\frac{\partial u_{0}}{\partial x}+\frac{1}{2}\left(\frac{\partial w}{\partial x}\right)^{2}+z \frac{\partial \phi_{x}}{\partial x} \\
\varepsilon_{y y}=\frac{\partial v_{0}}{\partial y}+\frac{1}{2}\left(\frac{\partial w}{\partial y}\right)^{2}+z \frac{\partial \phi_{y}}{\partial y}+\frac{w}{R}, \\
\gamma_{x y}=\frac{\partial u_{0}}{\partial y}+\frac{\partial v_{0}}{\partial x}+z\left(\frac{\partial \phi_{x}}{\partial y}+\frac{\partial \phi_{y}}{\partial x}\right)+\frac{\partial w}{\partial x} \frac{\partial w}{\partial y} \\
\gamma_{y z}=\phi_{y}+\frac{\partial w}{\partial y}-\frac{v_{0}}{R} \\
\gamma_{z x}=\phi_{x}+\frac{\partial w}{\partial x}
\end{gathered}
$$

The constitutive relations of the panel in which the thermal effects due to temperature difference are considered can be written as

$$
\begin{aligned}
\left\{\begin{array}{c}
\sigma_{x x} \\
\sigma_{y y} \\
\sigma_{y z} \\
\sigma_{z x} \\
\sigma_{x y}
\end{array}\right\}= & \left\{\begin{array}{ccccc}
Q_{11} & Q_{12} & 0 & 0 & 0 \\
Q_{21} & Q_{22} & 0 & 0 & 0 \\
0 & 0 & Q_{44} & 0 & 0 \\
0 & 0 & 0 & Q_{55} & 0 \\
0 & 0 & 0 & 0 & Q_{66}
\end{array}\right\} \\
& \times\left\{\left\{\begin{array}{c}
\varepsilon_{x x} \\
\varepsilon_{y y} \\
\gamma_{y z} \\
\gamma_{z x} \\
\gamma_{x y}
\end{array}\right\}-\left\{\begin{array}{c}
\alpha_{x x} \\
\alpha_{y y} \\
0 \\
0 \\
2 \alpha_{x y}
\end{array}\right\} \Delta T\right\},
\end{aligned}
$$

where $Q_{i j}(i=1,2$ and $j=1,2 ; i=4,5,6$ and $j=4,5,6)$ are the elastic constants which can be expressed as

$$
\begin{gathered}
Q_{11}=Q_{22}=\frac{E(z)}{1-v(z)^{2}}, \\
Q_{12}=Q_{21}=\frac{\nu(z) E(z)}{1-v(z)^{2}}, \\
Q_{44}=Q_{55}=Q_{66}=\frac{E(z)}{2(1+v(z))} .
\end{gathered}
$$

The thermal expansion coefficient $\alpha$ can be given as

$$
\alpha_{x x}=\alpha_{y y}=\alpha, \quad \alpha_{x y}=0
$$


2.4. Equations of Motion. By using Hamilton's principle, the motion equations in terms of midplane displacements are obtained as follows [20]:

$$
\begin{aligned}
\left(A_{11} I_{2}-B_{11} I_{1}\right) \frac{\partial^{2} u_{0}}{\partial x^{2}}+\left(A_{66} I_{2}-B_{66} I_{1}\right) \frac{\partial^{2} u_{0}}{\partial y^{2}} \\
+\left(A_{12} I_{2}+A_{66} I_{2}-B_{12} I_{1}-B_{66} I_{1}\right) \frac{\partial^{2} v_{0}}{\partial x \partial y} \\
+\left(B_{11} I_{2}-D_{11} I_{1}\right) \frac{\partial^{2} \phi_{x}}{\partial x^{2}}+\left(B_{66} I_{2}-D_{66} I_{1}\right) \frac{\partial^{2} \phi_{x}}{\partial y^{2}} \\
+\left(B_{12} I_{2}+B_{66} I_{2}-D_{12} I_{1}-D_{66} I_{1}\right) \frac{\partial^{2} \phi_{y}}{\partial x \partial y} \\
+\left[\left(A_{12} I_{2}-B_{12} I_{1}\right) \frac{1}{R}+A_{55} K I_{1}\right] \frac{\partial w_{0}}{\partial x} \\
+\left(A_{11} I_{2}-B_{11} I_{1}\right) \frac{\partial w_{0}}{\partial x} \frac{\partial^{2} w_{0}}{\partial x^{2}} \\
+\left(A_{66} I_{2}-B_{66} I_{1}\right) \frac{\partial w_{0}}{\partial x} \frac{\partial^{2} w_{0}}{\partial y^{2}} \\
+\left(A_{12} I_{2}+A_{66} I_{2}-B_{12} I_{1}-B_{66} I_{1}\right) \frac{\partial w_{0}}{\partial y} \frac{\partial^{2} w_{0}}{\partial x \partial y} \\
+A_{55} K I_{1} \phi_{x}=\left(I_{0} I_{2}-I_{1}^{2}\right) \ddot{u}_{0},
\end{aligned}
$$$$
\left(A_{66} I_{2}-B_{66} I_{1}\right) \frac{\partial^{2} v_{0}}{\partial x^{2}}+\left(A_{22} I_{2}-B_{22} I_{1}\right) \frac{\partial^{2} v_{0}}{\partial y^{2}}
$$$$
+\left[\left(A_{21}+A_{66}\right) I_{2}-\left(B_{21}+B_{66}\right) I_{1}\right] \frac{\partial^{2} u_{0}}{\partial x \partial y}
$$$$
+\left(B_{66} I_{2}-D_{66} I_{1}\right) \frac{\partial^{2} \phi_{y}}{\partial x^{2}}+\left(B_{22} I_{2}-D_{22} I_{1}\right) \frac{\partial^{2} \phi_{y}}{\partial y^{2}}
$$$$
+\left[\left(B_{21^{\prime}}+B_{66}\right) I_{2}-\left(D_{21^{\prime}}+D_{66}\right) I_{1}\right] \frac{\partial^{2} \phi_{x}}{\partial x \partial y}
$$$$
+\left[\left(A_{22} I_{2}-B_{22} I_{1}\right) \frac{1}{R}+A_{44} K I_{1}\right] \frac{\partial w_{0}}{\partial y}
$$$$
+\left(A_{66} I_{2}-B_{66} I_{1}\right) \frac{\partial w_{0}}{\partial y} \frac{\partial^{2} w_{0}}{\partial x^{2}}
$$$$
+\left(A_{22} I_{2}-B_{2} I_{1}\right) \frac{\partial w_{0}}{\partial y} \frac{\partial^{2} w_{0}}{\partial y^{2}}
$$$$
+\left[\left(A_{21}+A_{66}\right) I_{2}-\left(B_{21}+B_{66}\right) I_{1}\right] \frac{\partial w_{0}}{\partial x} \frac{\partial^{2} w_{0}}{\partial x \partial y}
$$$$
+K A_{44} I_{1} \phi_{y}+I_{2} K \frac{A_{44}}{R}\left(\phi_{y}+\frac{\partial w}{\partial y}-\frac{v_{0}}{R}\right)
$$$$
-A_{44} K I_{1} \frac{v_{0}}{R}=\left(I_{2} I_{0}-I_{1}^{2}\right) \ddot{v}_{0} \text {, }
$$

$$
\begin{aligned}
& A_{21} \frac{\partial u_{0}}{\partial x} \frac{\partial^{2} w_{0}}{\partial y^{2}}+A_{11} \frac{\partial u_{0}}{\partial x} \frac{\partial^{2} w_{0}}{\partial x^{2}}+2 A_{66} \frac{\partial u_{0}}{\partial y} \frac{\partial^{2} w_{0}}{\partial x \partial y} \\
& +\left(A_{21}+A_{66}\right) \frac{\partial^{2} u_{0}}{\partial x \partial y} \frac{\partial w_{0}}{\partial y}+A_{11} \frac{\partial^{2} u_{0}}{\partial x^{2}} \frac{\partial w_{0}}{\partial x} \\
& +A_{66} \frac{\partial^{2} u_{0}}{\partial y^{2}} \frac{\partial w_{0}}{\partial x}+2 A_{66} \frac{\partial v_{0}}{\partial x} \frac{\partial^{2} w_{0}}{\partial x \partial y}+A_{22} \frac{\partial v_{0}}{\partial y} \frac{\partial^{2} w_{0}}{\partial y^{2}} \\
& +\frac{1}{R} A_{21} \frac{\partial u_{0}}{\partial x}+\frac{1}{R} A_{22} \frac{\partial v_{0}}{\partial y}+A_{12} \frac{\partial v_{0}}{\partial y} \frac{\partial^{2} w_{0}}{\partial x^{2}} \\
& +A_{22} \frac{\partial^{2} v_{0}}{\partial y^{2}} \frac{\partial w_{0}}{\partial y}+A_{66} \frac{\partial^{2} v_{0}}{\partial x^{2}} \frac{\partial w_{0}}{\partial y} \\
& +\left(A_{12}+A_{66}\right) \frac{\partial^{2} v_{0}}{\partial x \partial y} \frac{\partial w_{0}}{\partial x}+A_{21} \frac{w}{R} \frac{\partial^{2} w}{\partial x^{2}} \\
& +A_{55} K \frac{\partial^{2} w_{0}}{\partial x^{2}}+A_{44} K \frac{\partial^{2} w_{0}}{\partial y^{2}}+\frac{3}{2 R} A_{12}\left(\frac{\partial w}{\partial x}\right)^{2} \\
& +\frac{3}{2 R} A_{22}\left(\frac{\partial w}{\partial y}\right)^{2}+A_{22} \frac{w}{R} \frac{\partial^{2} w}{\partial y^{2}} \\
& +A_{22} \frac{w}{R^{2}}+2\left(A_{21}+2 A_{66}\right) \frac{\partial w_{0}}{\partial x} \frac{\partial^{2} w_{0}}{\partial x \partial y} \frac{\partial w_{0}}{\partial y} \\
& +\frac{3}{2} A_{22}\left(\frac{\partial w_{0}}{\partial y}\right)^{2} \frac{\partial^{2} w_{0}}{\partial y^{2}} \\
& +\left(\frac{1}{2} A_{21}+A_{66}\right)\left(\frac{\partial w_{0}}{\partial x}\right)^{2} \frac{\partial^{2} w_{0}}{\partial y^{2}} \\
& +\left(A_{66}+\frac{1}{2} A_{21}\right)\left(\frac{\partial w_{0}}{\partial y}\right)^{2} \frac{\partial^{2} w_{0}}{\partial x^{2}} \\
& +\frac{3}{2} A_{11}\left(\frac{\partial w_{0}}{\partial x}\right)^{2} \frac{\partial^{2} w_{0}}{\partial x^{2}} \\
& +\left(\frac{1}{R} B_{21}+A_{55} K\right) \frac{\partial \phi_{x}}{\partial x}+B_{21} \frac{\partial \phi_{x}}{\partial x} \frac{\partial^{2} w_{0}}{\partial y^{2}} \\
& +2 B_{66} \frac{\partial \phi_{x}}{\partial y} \frac{\partial^{2} w_{0}}{\partial x \partial y}+\left(B_{21}+B_{66}\right) \frac{\partial^{2} \phi_{x}}{\partial x \partial y} \frac{\partial w_{0}}{\partial y} \\
& +B_{11} \frac{\partial^{2} \phi_{x}}{\partial x^{2}} \frac{\partial w_{0}}{\partial x}+B_{11} \frac{\partial \phi_{x}}{\partial x} \frac{\partial^{2} w_{0}}{\partial x^{2}} \\
& +B_{66} \frac{\partial^{2} \phi_{x}}{\partial y^{2}} \frac{\partial w_{0}}{\partial x}+\left(\frac{1}{R} B_{22}+A_{44} K\right) \frac{\partial \phi_{y}}{\partial y} \\
& +B_{22} \frac{\partial \phi_{y}}{\partial y} \frac{\partial^{2} w_{0}}{\partial y^{2}}+2 B_{66} \frac{\partial \phi_{y}}{\partial x} \frac{\partial^{2} w_{0}}{\partial x \partial y}+B_{66} \frac{\partial^{2} \phi_{y}}{\partial x^{2}} \frac{\partial w_{0}}{\partial y} \\
& +B_{12} \frac{\partial \phi_{y}}{\partial y} \frac{\partial^{2} w_{0}}{\partial x^{2}}+B_{22} \frac{\partial^{2} \phi_{y}}{\partial y^{2}} \frac{\partial w_{0}}{\partial y} \\
& +\left(B_{12}+B_{66}\right) \frac{\partial^{2} \phi_{y}}{\partial x \partial y} \frac{\partial w_{0}}{\partial x}
\end{aligned}
$$




$$
\begin{aligned}
& +N_{x x}^{T} \frac{\partial^{2} w_{0}}{\partial x^{2}}+N_{y y}^{T} \frac{\partial^{2} w_{0}}{\partial y^{2}}-K \frac{A_{44}}{R} \frac{\partial v_{0}}{\partial y}+\frac{N_{y y}^{T}}{R} \\
& -P^{\prime} \frac{\partial^{2} w_{0}}{\partial x^{2}}+\Delta P-\mu \dot{w}_{0}=I_{0} \ddot{w}_{0}, \\
& \left(A_{11} I_{1}-B_{11} I_{0}\right) \frac{\partial^{2} u_{0}}{\partial x^{2}}+\left(A_{66} I_{1}-B_{66} I_{0}\right) \frac{\partial^{2} u_{0}}{\partial y^{2}} \\
& +\left(A_{12} I_{1}+A_{66} I_{1}-B_{12} I_{0}-B_{66} I_{0}\right) \frac{\partial^{2} v_{0}}{\partial x \partial y} \\
& +\left(B_{11} I_{1}-D_{11} I_{0}\right) \frac{\partial^{2} \phi_{x}}{\partial x^{2}}+\left(B_{66} I_{1}-D_{66} I_{0}\right) \frac{\partial^{2} \phi_{x}}{\partial y^{2}} \\
& +\left(B_{12} I_{1}+B_{66} I_{1}-D_{12} I_{0}-D_{66} I_{0}\right) \frac{\partial^{2} \phi_{y}}{\partial x \partial y} \\
& +\left[\left(A_{12} I_{1}-B_{12} I_{0}\right) \frac{1}{R}+A_{55} K I_{0}\right] \frac{\partial w_{0}}{\partial x} \\
& +\left(A_{11} I_{1}-B_{11} I_{0}\right) \frac{\partial w_{0}}{\partial x} \frac{\partial^{2} w_{0}}{\partial x^{2}} \\
& +\left(A_{66} I_{1}-B_{66} I_{0}\right) \frac{\partial w_{0}}{\partial x} \frac{\partial^{2} w_{0}}{\partial y^{2}} \\
& +\left(A_{12} I_{1}+A_{66} I_{1}-B_{12} I_{0}-B_{66} I_{0}\right) \frac{\partial w_{0}}{\partial y} \frac{\partial^{2} w_{0}}{\partial x \partial y} \\
& +A_{55} K I_{0} \phi_{x}=\left(-I_{0} I_{2}+I_{1}^{2}\right) \ddot{\phi}_{x}, \\
& \left(A_{66} I_{1}-B_{66} I_{0}\right) \frac{\partial^{2} v_{0}}{\partial x^{2}}+\left(A_{22} I_{1}-B_{22} I_{0}\right) \frac{\partial^{2} v_{0}}{\partial y^{2}} \\
& +\left[\left(A_{21}+A_{66}\right) I_{1}-\left(B_{21}+B_{66}\right) I_{0}\right] \frac{\partial^{2} u_{0}}{\partial x \partial y} \\
& +\left(B_{66} I_{1}-D_{66} I_{0}\right) \frac{\partial^{2} \phi_{y}}{\partial x^{2}}+\left(B_{22} I_{1}-D_{22} I_{0}\right) \frac{\partial^{2} \phi_{y}}{\partial y^{2}} \\
& +\left[\left(B_{21^{\prime}}+B_{66}\right) I_{1}-\left(D_{21^{\prime}}+D_{66}\right) I_{0}\right] \frac{\partial^{2} \phi_{x}}{\partial x \partial y} \\
& +\left[\left(A_{22} I_{1}-B_{22} I_{0}\right) \frac{1}{R}+A_{44} K I_{0}\right] \frac{\partial w_{0}}{\partial y} \\
& +\left(A_{66} I_{1}-B_{66} I_{0}\right) \frac{\partial w_{0}}{\partial y} \frac{\partial^{2} w_{0}}{\partial x^{2}} \\
& +\left(A_{22} I_{1}-B_{2} I_{0}\right) \frac{\partial w_{0}}{\partial y} \frac{\partial^{2} w_{0}}{\partial y^{2}} \\
& +\left[\left(A_{21}+A_{66}\right) I_{1}-\left(B_{21}+B_{66}\right) I_{0}\right] \frac{\partial w_{0}}{\partial x} \frac{\partial^{2} w_{0}}{\partial x \partial y}
\end{aligned}
$$

$$
\begin{aligned}
& +A_{44} K I_{0} \phi_{y}-I_{0} A_{44} K \frac{v_{0}}{R} \\
& +I_{1} \frac{A_{44}}{R} K\left(\phi_{y}+\frac{\partial w}{\partial y}-\frac{v_{0}}{R}\right)=\left(-I_{2} I_{0}+I_{1}^{2}\right) \ddot{\phi}_{y}
\end{aligned}
$$

where $\mu$ is the damping coefficient, all kinds of the stiffness elements $A_{i j}, B_{i j}$, and $D_{i j}$ of the FGM cylindrical panel are denoted by

$$
\begin{gathered}
\left(A_{i j}, B_{i j}, D_{i j}\right)=\int_{-h / 2}^{h / 2} Q_{i j}\left(1, z, z^{2}\right) d z, \quad i, j=1,2,6, \\
A_{i j}=\int_{-h / 2}^{h / 2} Q_{i j} d z, \quad i, j=4,5,
\end{gathered}
$$

and various mass inertia terms $I_{i}$ in (12) can be defined as

$$
I_{i}=\int_{-h / 2}^{h / 2} z^{i} p(z) d z, \quad i=0,1,2 .
$$

Thermal force resultants due to temperature rise are functions of the temperature and coefficient of thermal expansion equation. They can be calculated by

$$
\left\{\begin{array}{c}
N_{x x}^{T} \\
N_{y y}^{T}
\end{array}\right\}=-\int_{-h / 2}^{h / 2}\left[\begin{array}{lll}
Q_{11} & Q_{12} & 0 \\
Q_{21} & Q_{22} & 0
\end{array}\right]\left\{\begin{array}{c}
\alpha_{x x} \\
\alpha_{y y} \\
0
\end{array}\right\} \Delta T d z .
$$

Here, the shear correction factor $K$ that is introduced by Reddy [25] and Kadoli and Ganesan [26] is equal to 5/6.

For simply supported hypersonic FGM circular cylindrical panel with rectangular base, the first two mode shapes that satisfy the boundary conditions are assumed to be

$$
\begin{aligned}
u_{0}= & u_{1}(t) \cos \left(\frac{\pi x}{L}\right) \sin \left(\frac{3 \pi y}{b}\right) \\
& +u_{2}(t) \cos \left(\frac{3 \pi x}{L}\right) \sin \left(\frac{\pi y}{b}\right), \\
v_{0}= & v_{1}(t) \sin \left(\frac{\pi x}{L}\right) \cos \left(\frac{3 \pi y}{b}\right) \\
& +v_{2}(t) \sin \left(\frac{3 \pi x}{L}\right) \cos \left(\frac{\pi y}{b}\right), \\
w_{0}= & w_{1}(t) \sin \left(\frac{\pi x}{L}\right) \sin \left(\frac{3 \pi y}{b}\right) \\
& +w_{2}(t) \sin \left(\frac{3 \pi x}{L}\right) \sin \left(\frac{\pi y}{b}\right), \\
\phi_{0}= & \phi_{1}(t) \cos \left(\frac{\pi x}{L}\right) \sin \left(\frac{3 \pi y}{b}\right) \\
& +\phi_{2}(t) \cos \left(\frac{3 \pi x}{L}\right) \sin \left(\frac{\pi y}{b}\right), \\
\psi_{0}= & \psi_{1}(t) \sin \left(\frac{\pi x}{L}\right) \cos \left(\frac{3 \pi y}{b}\right) \\
& +\psi_{2}(t) \sin \left(\frac{3 \pi x}{L}\right) \cos \left(\frac{\pi y}{b}\right),
\end{aligned}
$$


where $u_{1}, v_{1}, w_{1}, \phi_{1}, \psi_{1}$ and $u_{2}, v_{2}, w_{2}, \phi_{2}, \psi_{2}$ are the time dependence amplitudes of the first two modes. The constant $b$ can be represented by $b=R \theta$.

Compared to the transverse inertia term, the influences of the in-plane and rotary inertia terms on the vibration of the panel are small and can be neglected; see [27, 28]. Following the work given in [20], the displacement components of $u_{0}, v_{0}, \phi_{x}$, and $\phi_{y}$ can be expressed in terms of $w_{0}$. Applying the Galerkin method on (12), one can obtain a coupled set of nonlinear ordinary differential equations in time that can take the form as follows:

$$
\begin{aligned}
\ddot{w}_{1}+ & c_{1} \dot{w}_{1}+\eta_{101} w_{1}+P_{1}^{\prime} \cos \left(\omega_{1} t\right) w_{1}+g_{102} w_{1}^{2} \\
& +g_{103} w_{1} w_{2}+g_{104} w_{2}^{2}+g_{105} w_{1}^{3}+g_{106} w_{1}^{2} w_{2} \\
& +g_{107} w_{2}^{2} w_{1}+g_{108} w_{2}^{3}-\Delta p_{1}=0, \\
\ddot{w}_{2}+ & c_{2} \dot{w}_{2}+\eta_{201} w_{2}+P_{2}^{\prime} \cos \left(\omega_{1} t\right) w_{2}+g_{202} w_{1}^{2} \\
& +g_{203} w_{1} w_{2}+g_{204} w_{2}^{2}+g_{205} w_{1}^{3}+g_{206} w_{1}^{2} w_{2} \\
& +g_{207} w_{2}^{3}+g_{208} w_{2}^{2} w_{1}-\Delta p_{2}=0,
\end{aligned}
$$

where $\eta_{101}=g_{101}+N_{1}^{\prime}$ and $\eta_{201}=g_{201}+N_{2}^{\prime}$. The coefficients $N_{1}^{\prime}$ and $N_{2}^{\prime}$ are the results of thermal stress resultants and the static components of in-plane preloads. The variables $\Delta p_{1}$ and $\Delta p_{2}$ are related to the aerodynamic pressure; they can be expressed as

$$
\begin{aligned}
& \Delta p_{1} \\
& =M_{a}\left(C_{m 111} w_{1}+C_{m 112} w_{2}\right) \\
& +M_{a}^{2}\left(C_{m 121} w_{1}^{2}+C_{m 122} w_{1} w_{2}+C_{m 123} w_{2}^{2}\right) \\
& +M_{a}^{3}\left(C_{m 131} w_{1}^{3}+C_{m 132} w_{1}^{2} w_{2}+C_{m 133} w_{1} w_{2}^{2}+C_{m 134} w_{2}^{3}\right) \\
& +D_{m 101} \dot{w}_{1}^{3}+D_{m 102} \dot{w}_{2}^{3}+D_{m 103} \dot{w}_{1}^{2} \dot{w}_{2} \\
& +D_{m 104} \dot{w}_{1} \dot{w}_{2}^{2}+D_{m 105} \dot{w}_{1}^{2} \\
& +D_{m 106} \dot{w}_{2}^{2}+D_{m 107} \dot{w}_{1} \dot{w}_{2}+D_{m 108} \dot{w}_{1} \\
& +M_{a}\left[\left(D_{m 111} w_{1}+D_{m 112} w_{2}\right) \dot{w}_{1}\right. \\
& \quad+\left(D_{m 113} w_{1}+D_{m 114} w_{2}\right) \dot{w}_{2} \\
& \quad+\left(D_{m 115} w_{1}+D_{m 116} w_{2}\right) \dot{w}_{1}^{2} \\
& \quad+\left(D_{m 117} w_{1}+D_{m 118} w_{2}\right) \dot{w}_{2}^{2} \\
& \left.+\left(D_{m 119} w_{1}+D_{m 1110} w_{2}\right) \dot{w}_{1} \dot{w}_{2}\right] \\
& +M_{a}^{2}\left[\left(D_{m 121} w_{1}^{2}+D_{m 122} w_{1} w_{2}+D_{m 123} w_{2}^{2}\right) \dot{w}_{1}\right. \\
& \left.+\left(D_{m 124} w_{1}^{2}+D_{m 125} w_{1} w_{2}+D_{m 126} w_{2}^{2}\right) \dot{w}_{2}\right]
\end{aligned}
$$

$$
\begin{aligned}
& \Delta p_{2} \\
& =M_{a}\left(C_{m 211} w_{1}+C_{m 212} w_{2}\right) \\
& +M_{a}^{2}\left(C_{m 221} w_{1}^{2}+C_{m 222} w_{1} w_{2}+C_{m 223} w_{2}^{2}\right) \\
& +M_{a}^{3}\left(C_{m 231} w_{1}^{3}+C_{m 232} w_{1}^{2} w_{2}+C_{m 233} w_{1} w_{2}^{2}+C_{m 234} w_{2}^{3}\right) \\
& +D_{m 201} \dot{w}_{1}^{3}+D_{m 202} \dot{w}_{2}^{3}+D_{m 203} \dot{w}_{1}^{2} \dot{w}_{2}+D_{m 204} \dot{w}_{1} \dot{w}_{2}^{2} \\
& +D_{m 205} \dot{w}_{1}^{2}+D_{m 206} \dot{w}_{2}^{2}+D_{m 207} \dot{w}_{1} \dot{w}_{2}+D_{m 208} \dot{w}_{2} \\
& +M_{a}\left[\left(D_{m 211} w_{1}+D_{m 212} w_{2}\right) \dot{w}_{1}\right. \\
& \quad+\left(D_{m 213} w_{1}+D_{m 214} w_{2}\right) \dot{w}_{2} \\
& \quad+\left(D_{m 215} w_{1}+D_{m 216} w_{2}\right) \dot{w}_{1}^{2} \\
& \quad+\left(D_{m 217} w_{1}+D_{m 218} w_{2}\right) \dot{w}_{2}^{2} \\
& \left.+\left(D_{m 219} w_{1}+D_{m 2110} w_{2}\right) \dot{w}_{1} \dot{w}_{2}\right] \\
& +M_{a}^{2}\left[\left(D_{m 221} w_{1}^{2}+D_{m 222} w_{1} w_{2}+D_{m 223} w_{2}^{2}\right) \dot{w}_{1}\right. \\
& \left.+\left(D_{m 224} w_{1}^{2}+D_{m 225} w_{1} w_{2}+D_{m 226} w_{2}^{2}\right) \dot{w}_{2}\right]
\end{aligned}
$$

All the coefficients in (18a) and (18b) can be affected by geometric and physical parameters and temperature field of the panel. And the coefficients in (19a) and (19b) are dependent on geometric and free-stream air density and freestream speed of sound. They are too long to be listed out in the paper for abbreviation. This is a nonlinear dynamic system which includes cubic and quadratic terms. To study the nonlinear aeroelastic behavior of the FGM cylindrical panel in hypersonic flow, the obtained nonlinear ordinary differential equations can be solved by the fourth-order Runge-Kutta method.

\section{Numerical Results and Discussions}

In order to validate the numerical results presented in this study, a comparison is shown in Figure 2 for a simply supported intact aluminum-zirconia FGM square plate with length $0.2 \mathrm{~m}$ and thickness $0.01 \mathrm{~m}$. A suddenly uniform transverse load of intensity of $q_{0}=1 \mathrm{MPa}$ is applied on it. The material properties vary in the thickness by following a simple power law and the power law exponent is taken as $n=0.2$. It means that the plate is zirconia-rich on the top surface and aluminum-rich at the bottom surface. In Figure 2, temporal evolution curve of center deflection of the square plate is given by Reddy's finite element results (see [29]) and the results of this study. The dimensionless time and center deflection are defined as $\bar{t}=t \sqrt{E_{b} /\left(\rho_{b} a^{2}\right)}$ and $\bar{w}=w_{c} E_{b} h /\left(q_{0} a^{2}\right)$, respectively. According to this figure, close agreements between present methodology and Reddy's finite element results can be observed. Although the plate is subjected to the aerodynamic force in this study and not uniform transverse load, as external pressures, both of them play the same role on the plate. 


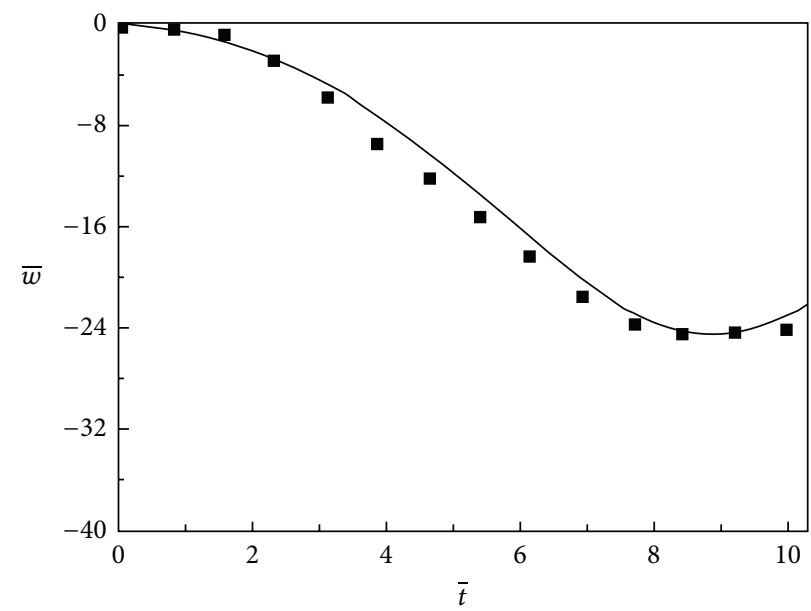

FIGURE 2: Comparison of nondimensional center deflection of the square plate with simply supported edges under a suddenly applied uniform load by Reddy's FEM results [29] and present (—: present; $\square$ : Reddy's).

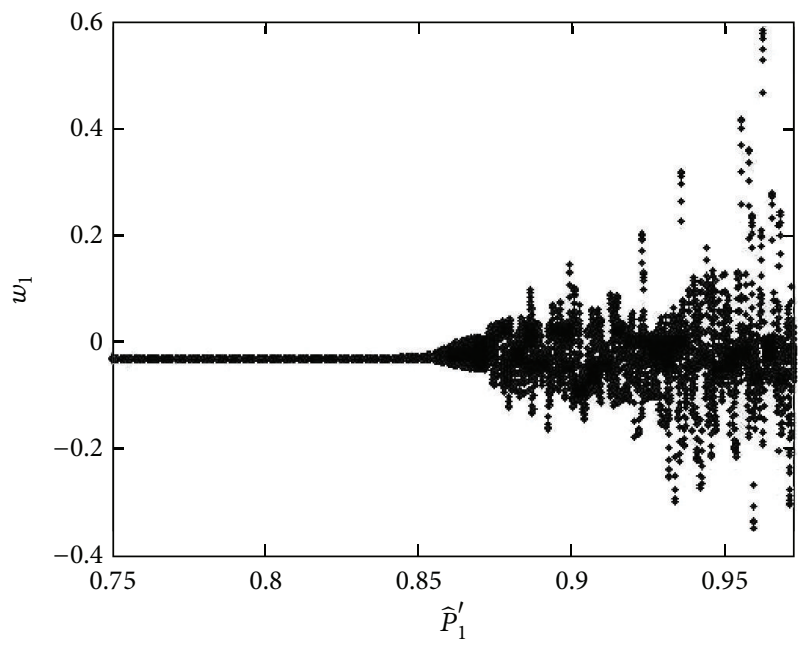

(a)

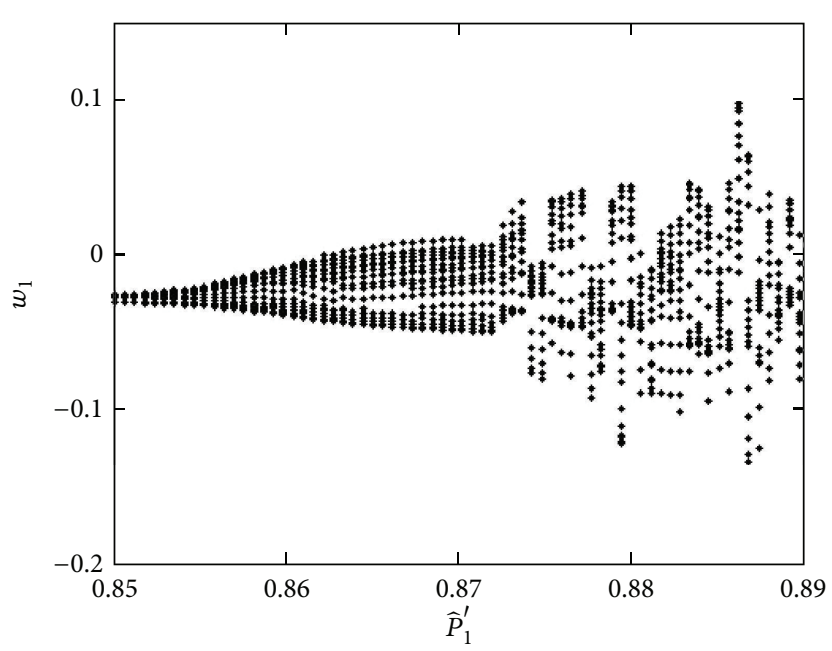

(c)

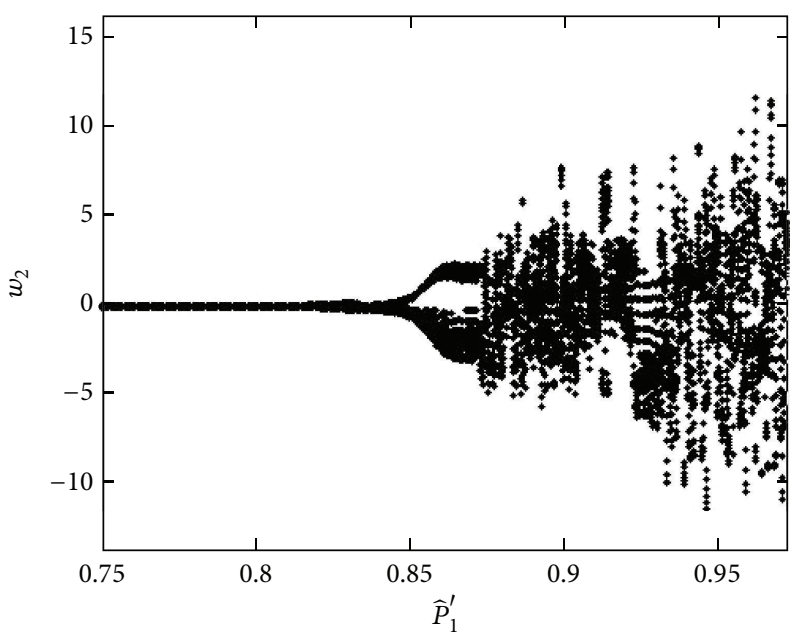

(b)

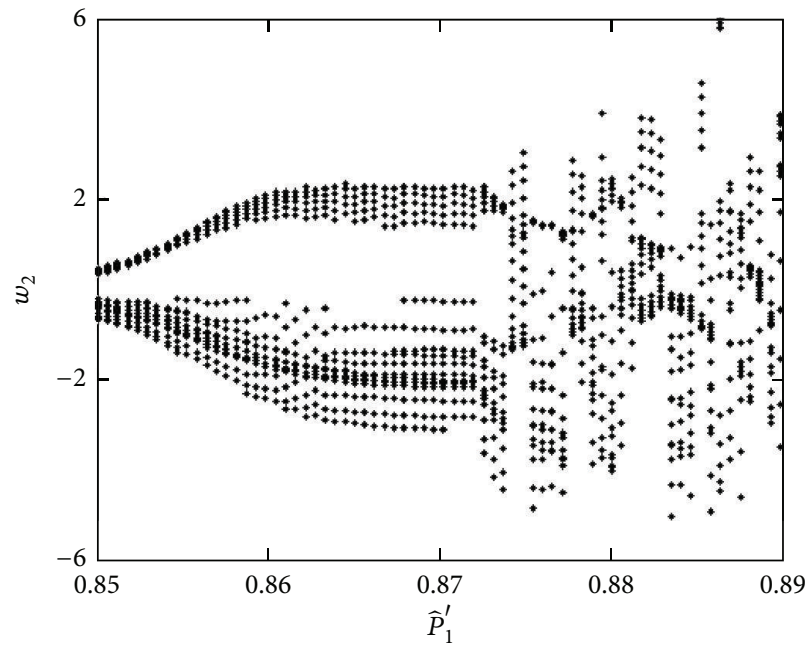

(d)

Figure 3: (a) and (b) depict the bifurcation diagrams of $P_{1}^{\prime}$ versus the first two modes $w_{1}$ and $w_{2}$ which are given when the volume fraction index is taken to be 0.5. The Mach number is taken to be 5.0. The magnitude and frequency of the in-plane excitation are taken to be $P_{1}^{\prime} \in$ $\left[5.5 \times 10^{7}, 7.79 \times 10^{7}\right]$ and $161 \mathrm{~Hz}$, respectively. (c) and (d) are partially enlarged details for (a) and (b), respectively. The value of horizontal coordinate is defined as $\widehat{P}_{1}^{\prime}=P_{1}^{\prime} / 7.79 \times 10^{7}$. 


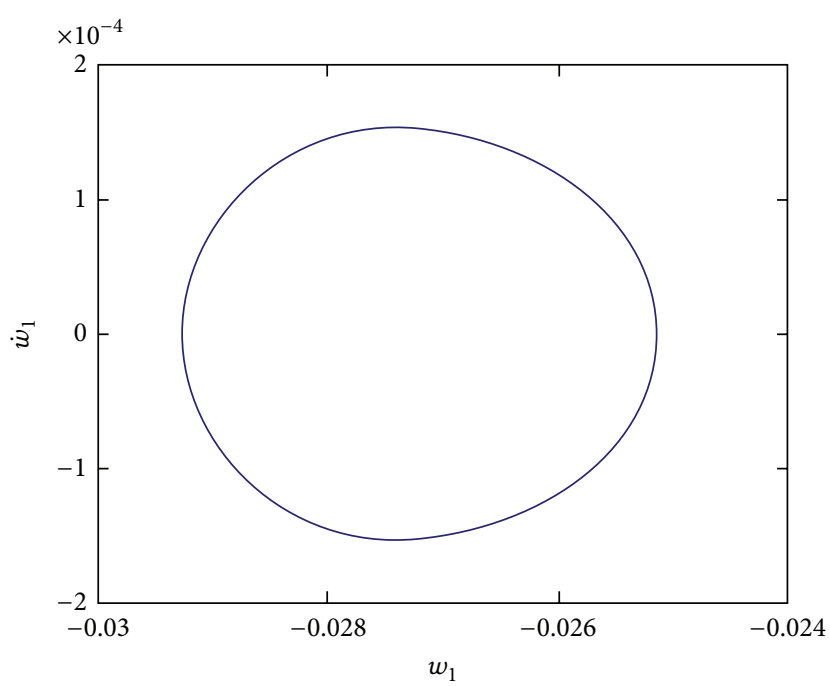

(a)

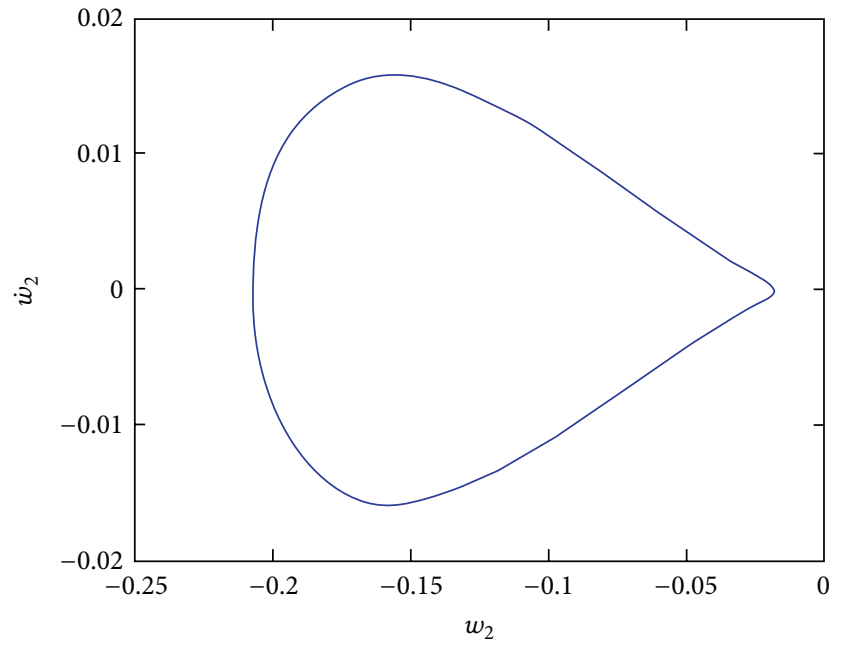

(c)

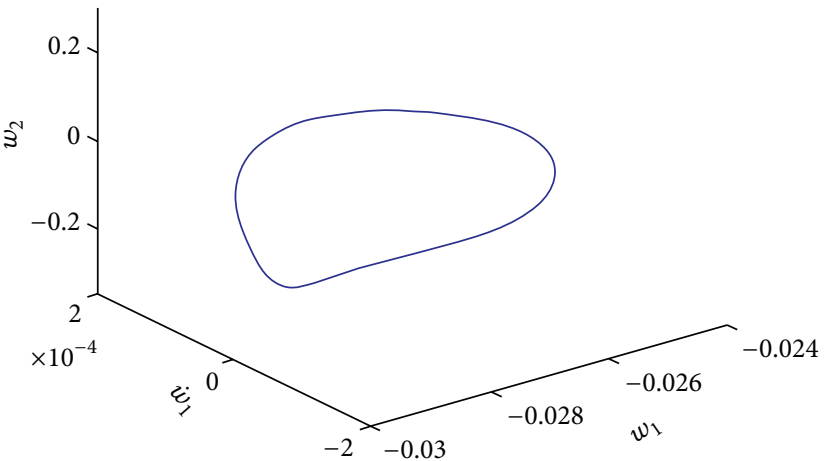

(e)

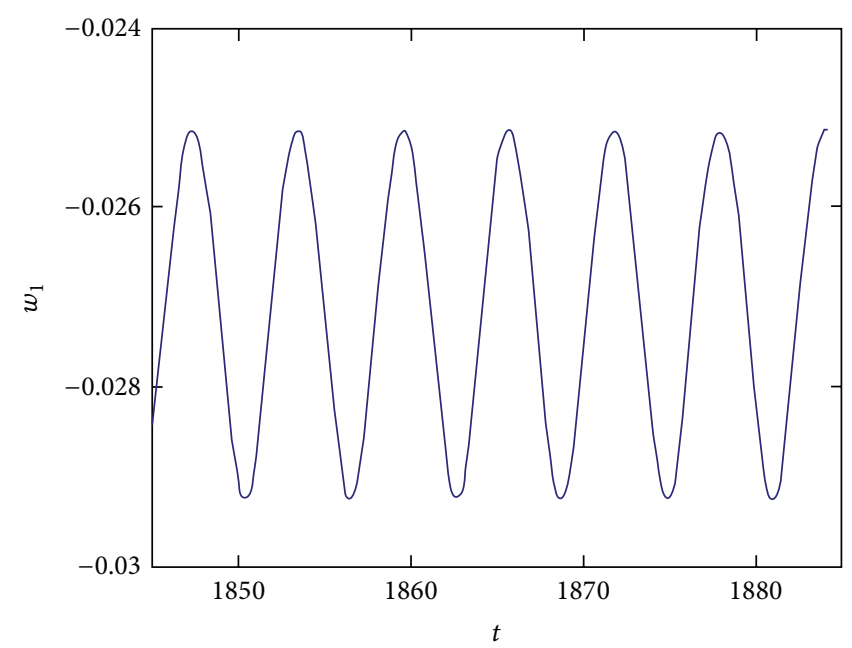

(b)

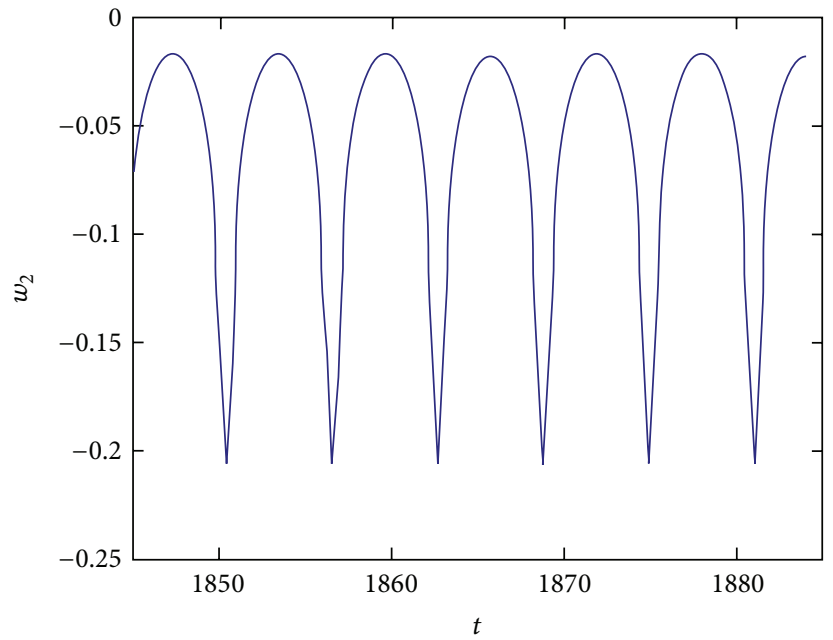

(d)

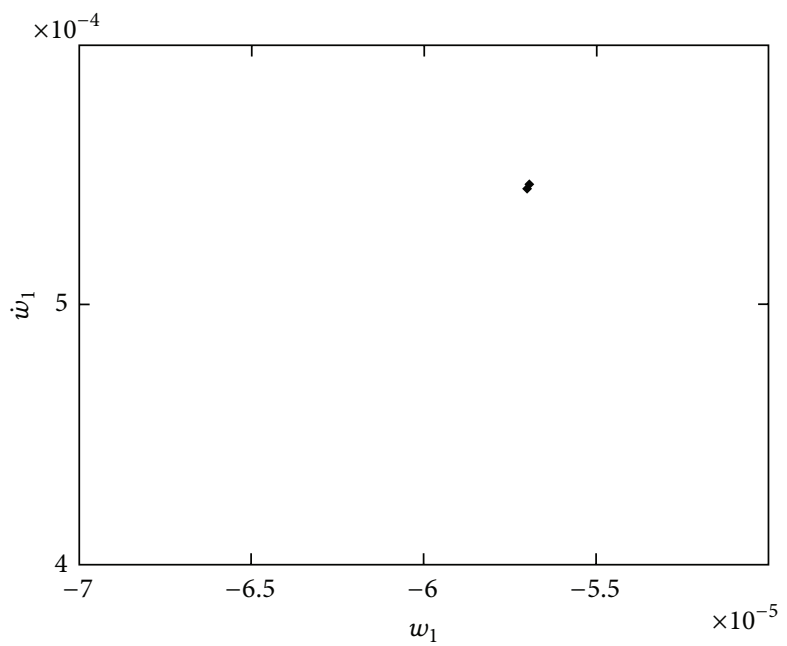

(f)

FIGURE 4: The periodic response of the FGM cylindrical panel occurs when the magnitude of the in-plane excitation is taken to be $5.85 \times 10^{7}$. The volume fraction index is taken to be 0.5. The Mach number is taken to be 5.0. (a) The phase portrait on plane $\left(w_{1}, \dot{w}_{1}\right)$; $(\mathrm{b})$ the time response on the planes $\left(t, w_{1}\right)$; (c) the phase portrait on plane $\left(w_{2}, \dot{w}_{2}\right)$; (d) the responses on the planes $\left(t, w_{2}\right)$; (e) three-dimensional phase portrait in space $\left(w_{1}, \dot{w}_{1}, w_{2}\right) ;(f)$ the Poincare section on plane $\left(w_{1}, \dot{w}_{1}\right)$. 


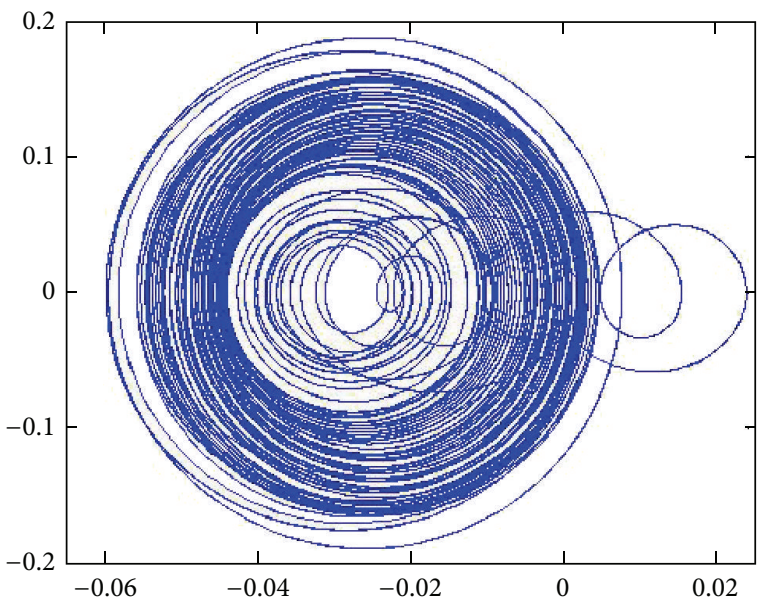

(a)

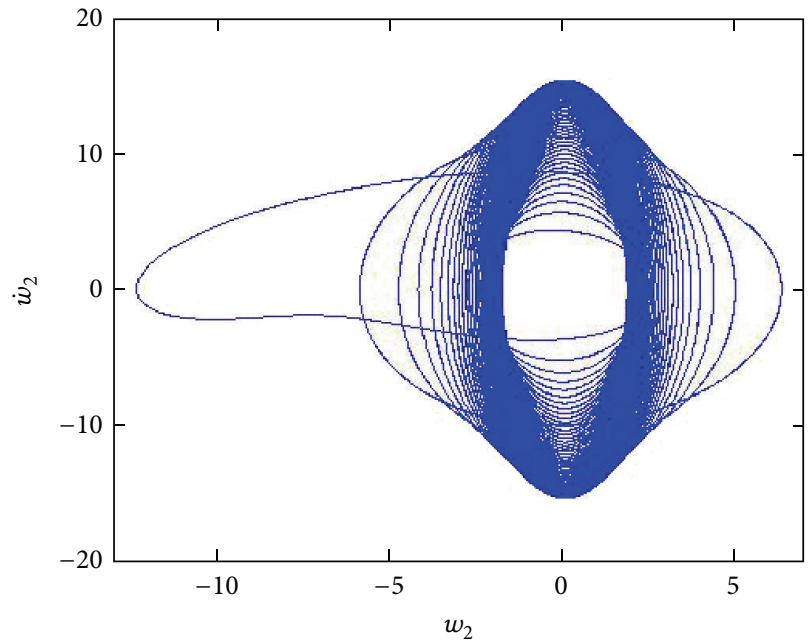

(c)

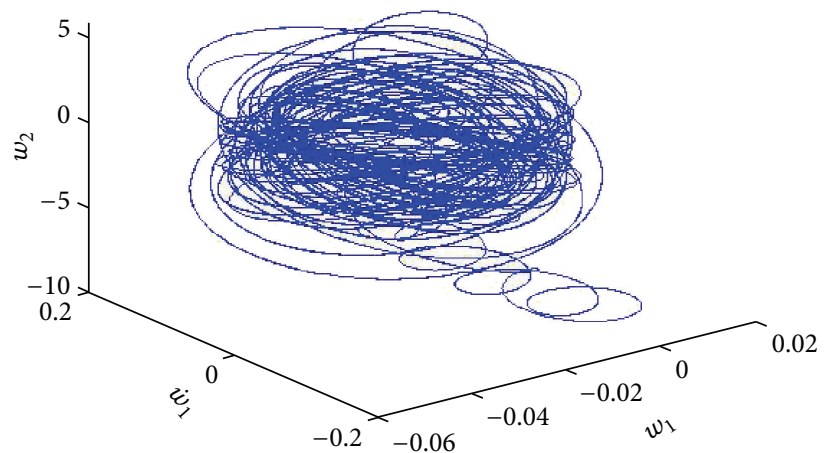

(e)

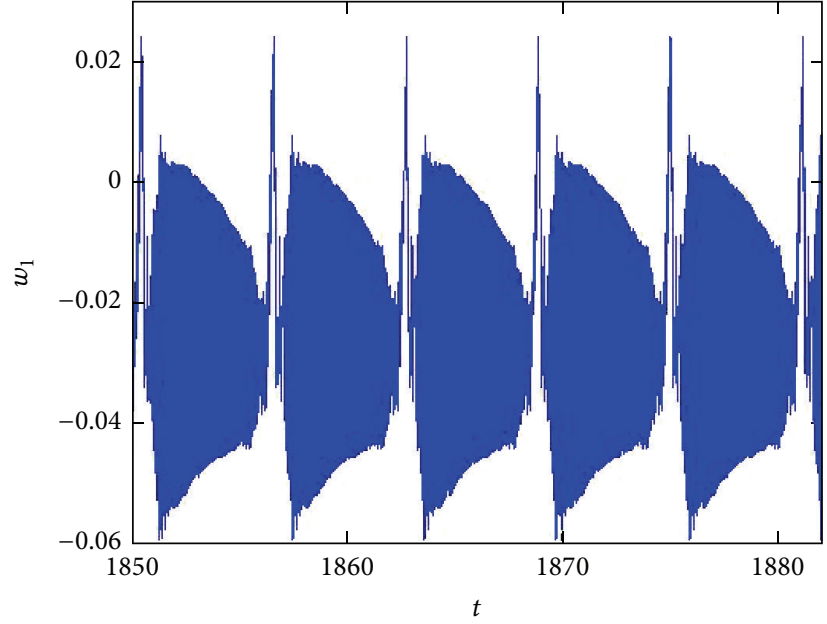

(b)

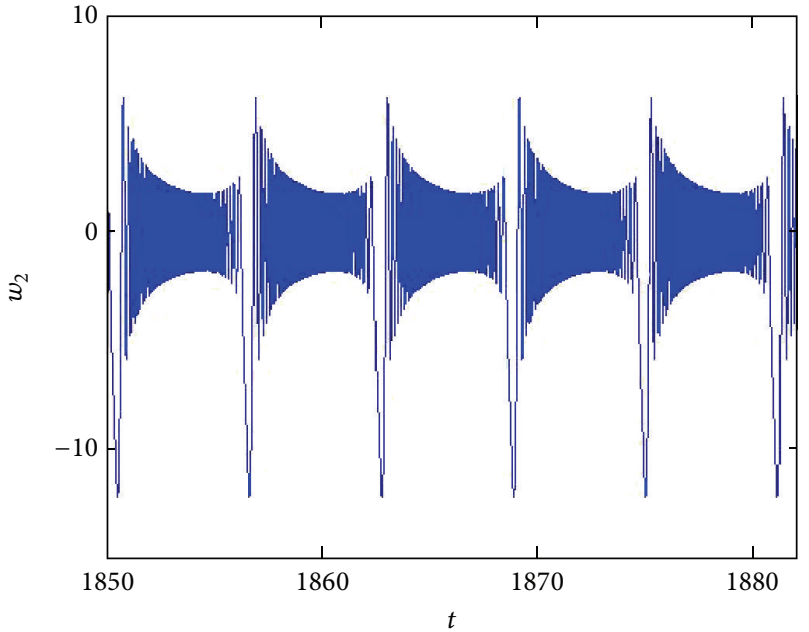

(d)

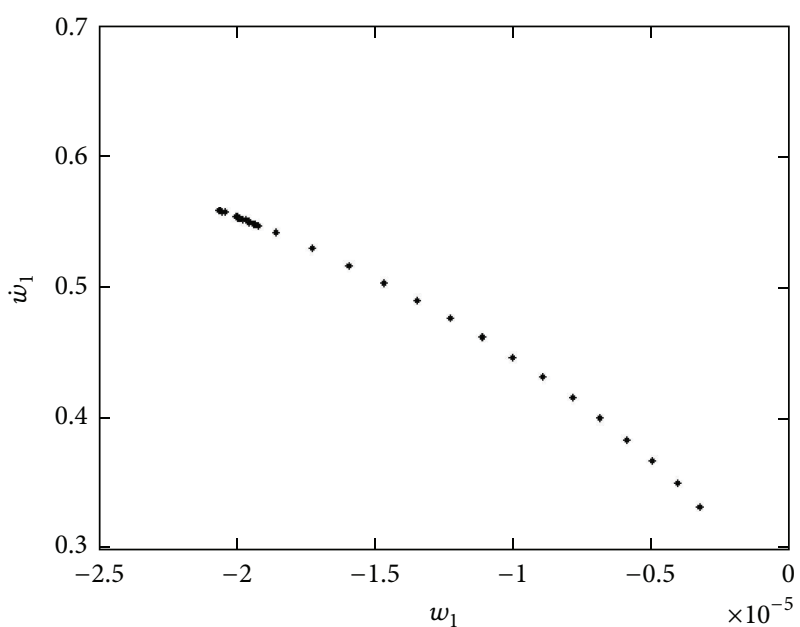

(f)

FIGURE 5: The multiple-periodic motion of the FGM cylindrical panel occurs when the magnitude of the in-plane excitation is taken to be $6.70 \times 10^{7}$. The volume fraction index is taken to be 0.5 . The Mach number is taken to be 5.0 . 


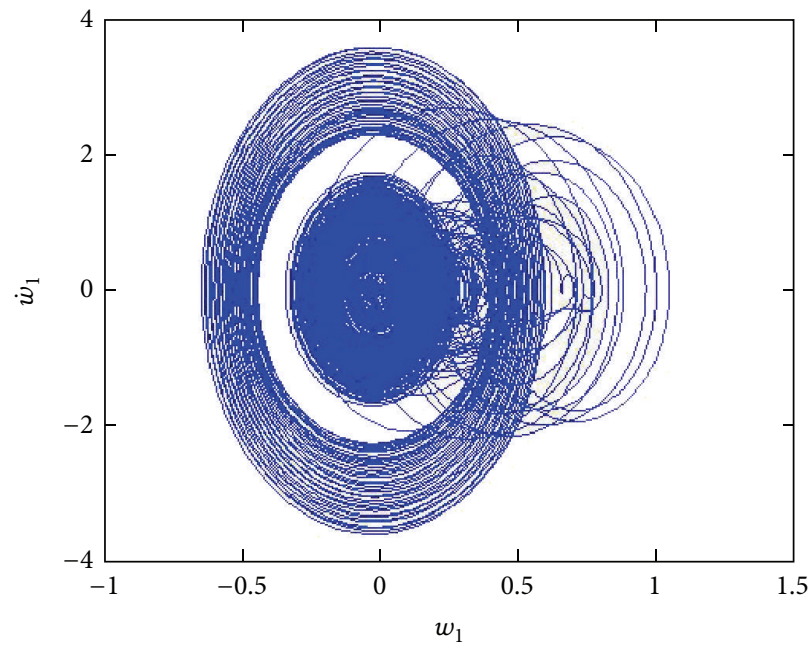

(a)

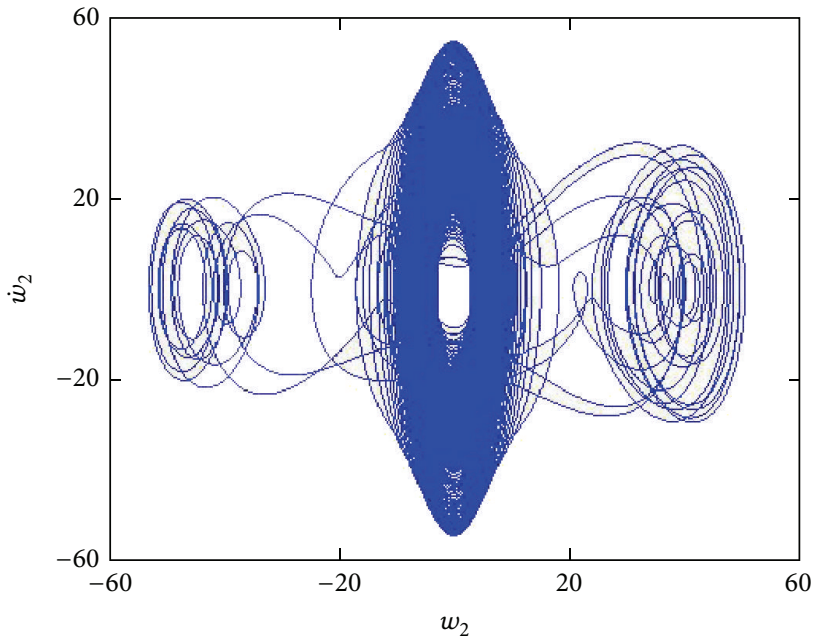

(c)

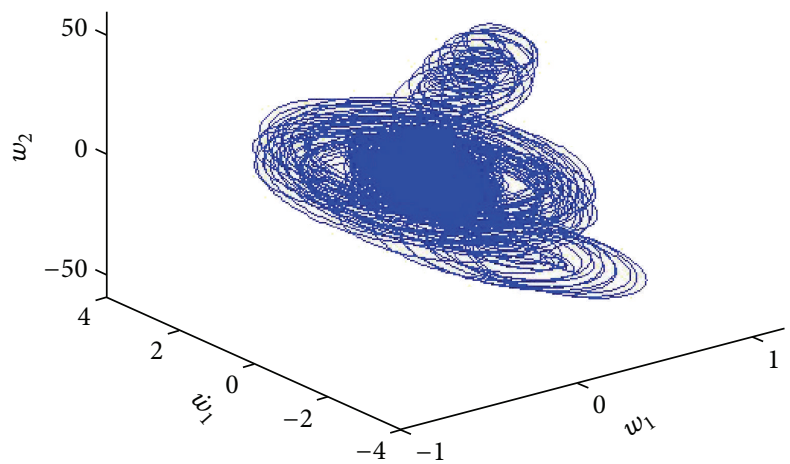

(e)

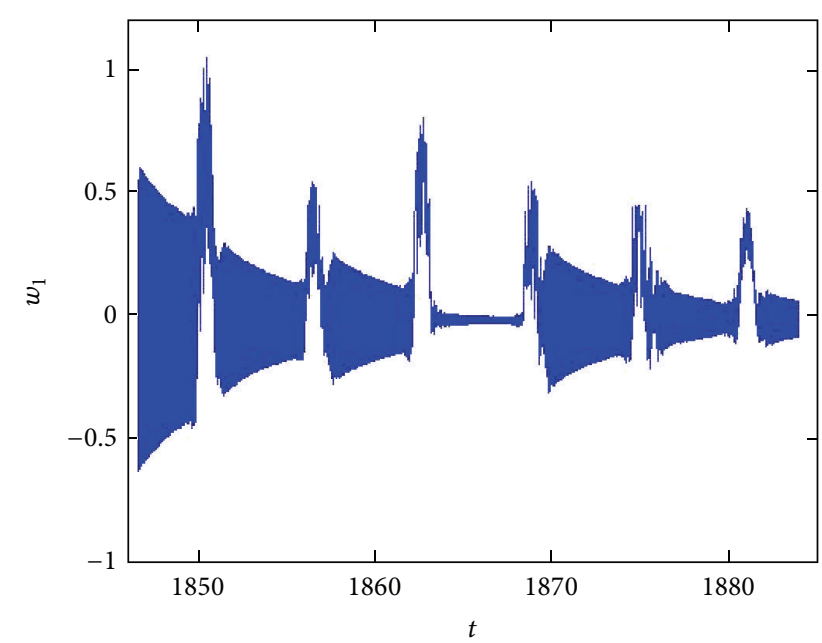

(b)

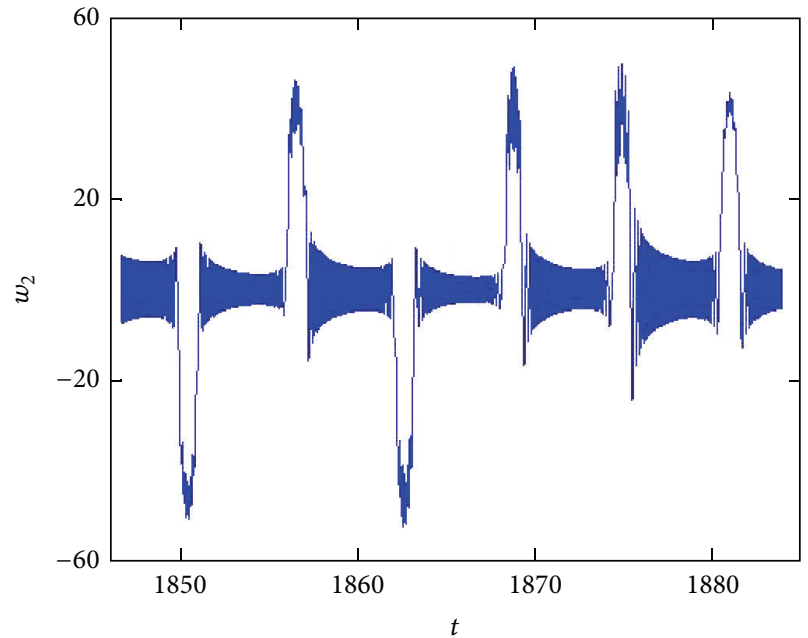

(d)

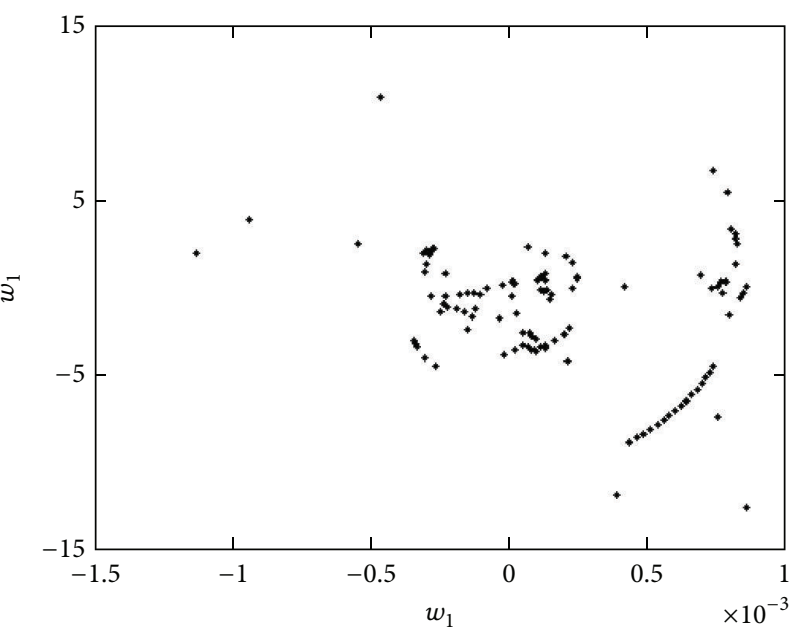

(f)

FIGURE 6: The chaotic motion of the FGM cylindrical panel occurs when the magnitude of the in-plane excitation is taken to be $7.79 \times 10^{7}$. The volume fraction index is taken to be 0.5 . The Mach number is taken to be 5.0 . 


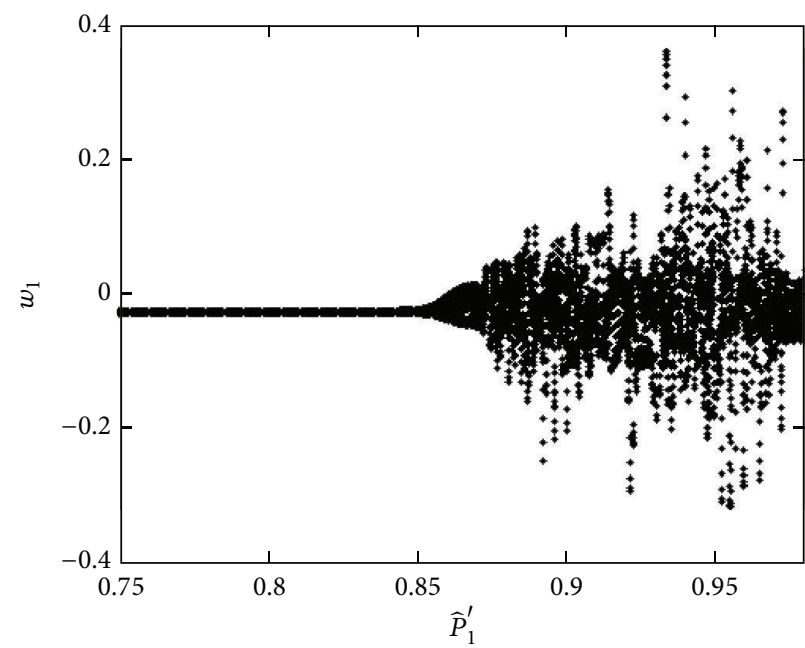

(a)

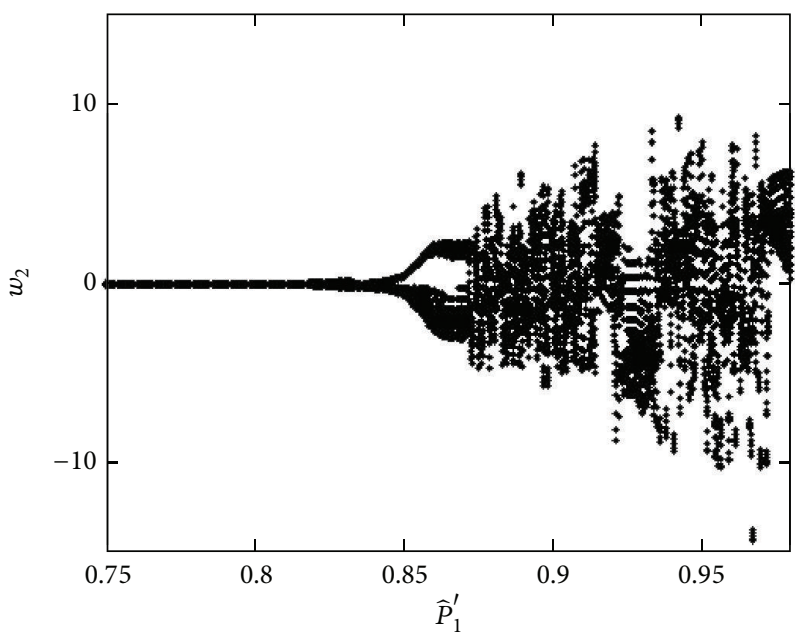

(b)

Figure 7: (a) and (b) depict the bifurcation diagrams of $P_{1}^{\prime}$ versus $w_{1}$ and $w_{2}$ which are given when the Mach number is taken to be 10.0. The value of horizontal coordinate has the same definition as that in Figure 3.

In this study, $\mathrm{Al}_{2} \mathrm{O}_{3}$ and $\mathrm{Ti}-6 \mathrm{Al}-4 \mathrm{~V}$ are chosen as the two constituent materials of the FGM cylindrical panel. The properties for these two constituent materials can be found in Shen [30]. For simplicity, it is assumed that Poisson's ratio is a constant as the value of $v=0.3$ and the reference temperature remained a constant as $300 \mathrm{~K}$. The simply supported FGM cylindrical panel has the following geometries: length $L=$ $2.4 \mathrm{~m}$, radius of curvature $R=1.5 \mathrm{~m}$, thickness $h=0.002 \mathrm{~m}$, and angular width $\theta=(\pi / 2) R$. In all of the numerical analyses, the temperature difference through the thickness of the panel was assumed to have attained a steady state $100 \mathrm{~K}$. In all of the calculations, the initial conditions are fixed at the value of $w_{1}=0.0004, \dot{w}_{1}=-0.00025, w_{2}=0.0005$, and $\dot{w}_{2}=0.00006$. In addition, the flow field characteristics are as follows: the free-stream air density $\rho_{\infty}=1.225 \mathrm{~kg} / \mathrm{m}^{3}$, the free-stream speed of sound $a_{\infty}=340.3 \mathrm{~m} / \mathrm{s}$, and the adiabatic exponent $\gamma=1.4$; see Librescu et al. [31]. In given conditions, the influences of the in-plane excitation and Mach numbers on the nonlinear dynamic responses of the FGM cylindrical panel in hypersonic flow are investigated. In order to perform analysis of the chaotic and periodic responses for the FGM cylindrical panel, the bifurcation diagrams are depicted. The dimensionless transverse amplitudes are defined as $\widetilde{w}=w / h$ here. To study convenience, the overbar is dropped in the following research.

Firstly, the Mach number and frequency of the in-plane excitation are taken to be 5.0 and $161 \mathrm{~Hz}$, respectively. And the volume fraction index is as $N=0.5$. Figure 3 plots the bifurcation diagrams of transverse amplitude of the first two modes by changing the in-plane loads with other parameters fixed. In Figures 3(a) and 3(b), the bifurcation diagrams of the $P_{1}^{\prime}\left(P_{1}^{\prime} \in\left[5.5 \times 10^{7}, 7.79 \times 10^{7}\right]\right)$ versus $w_{1}$ and $w_{2}$ are given. Chaotic and periodic motions have been detected, as indicated in Figure 3, which shows the very complex and rich nonlinear dynamics.

As the amplitude of excitation increases within region of $P_{1}^{\prime} \in\left[5.5 \times 10^{7}, 6.63 \times 10^{7}\right]$, periodic motion occurs for the FGM cylindrical panel. Furthermore, a region of multiperiodic motion exists when the amplitude of excitation varied from $6.63 \times 10^{7}$ to $6.80 \times 10^{7}$. The system appears to undergo complicated bifurcations where the system is in the instability region. Within the apparently chaotic region where the amplitude of excitation changes from $6.80 \times 10^{7}$ to $7.79 \times 10^{7}$, there are some regions where the response is periodic. Figures $3(\mathrm{c})$ and $3(\mathrm{~d})$ are partially enlarged details for Figures 3(a) and 3(b), respectively.

To better understand nonlinear dynamical behaviors, phase portraits, time responses, the Poincare section, and three-dimensional phase portrait are illustrated. Figure 4 illustrates nonlinear dynamic response for the FGM cylindrical panel when the forcing excitation is $5.85 \times 10^{7}$. The twodimensional phase plane portraits are given in Figures 4(a) and $4(\mathrm{c})$ by $\left(w_{j}, \dot{w}_{j}\right)$. Figures $4(\mathrm{~b})$ and $4(\mathrm{~d})$ denote the time responses on the planes $\left(t, w_{j}\right)$, respectively. Here subscript " $j$ " indicates the first two modes and takes the value $j=1,2$. Figures 4(e) and 4(f) represent the three-dimensional phase portrait in space $\left(w_{1}, \dot{w}_{1}, w_{2}\right)$ and the Poincare section on plane $\left(w_{1}, \dot{w}_{1}\right)$, respectively (see $\left.[20,32,33]\right)$. It is known that if the Poincare section displays a finite number of points, the motion can be periodic. Since there is single point in Poincare sections given in Figure 4(f), it can be concluded that there exists periodic oscillations motion for the FGM cylindrical panel in this case. Figure 5 suggests that the multiple-periodic motion of the FGM cylindrical panel occurs when the inplane excitationchanges to $6.70 \times 10^{7}$. Figure 6 illustrates the existence of the chaotic motion for this system when the forcing excitation is $7.79 \times 10^{7}$.

Figure 7, where in-plane excitation is also taken as the bifurcation parameter, shows the effect of in-plane excitation on the dynamics of the FGM cylindrical panel when the Mach is 10. Compared to the bifurcation diagrams given in Figure 3 whose Mach is 5, we can see that the appearance of the bifurcation point is almost in the same locations. In the instability region, the chaotic and multiperiodic motion may 


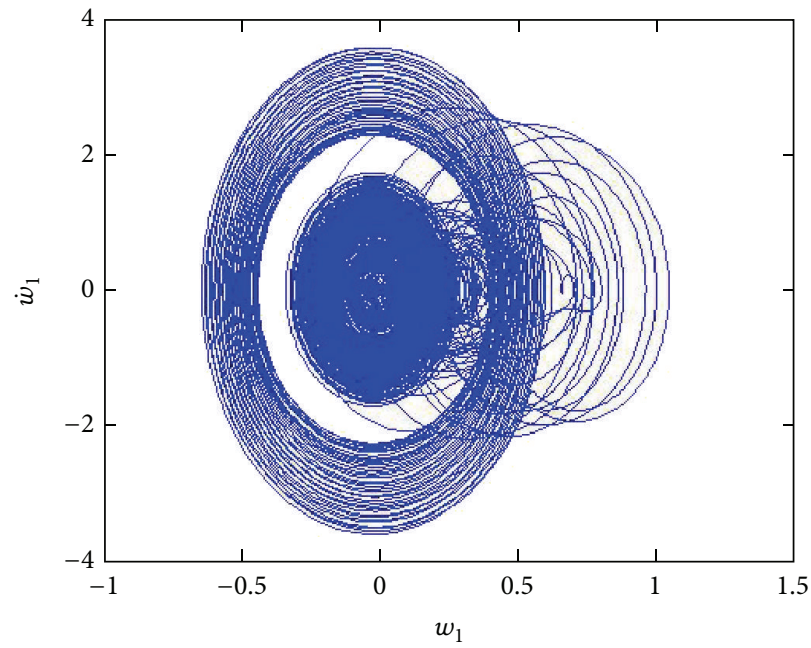

(a)

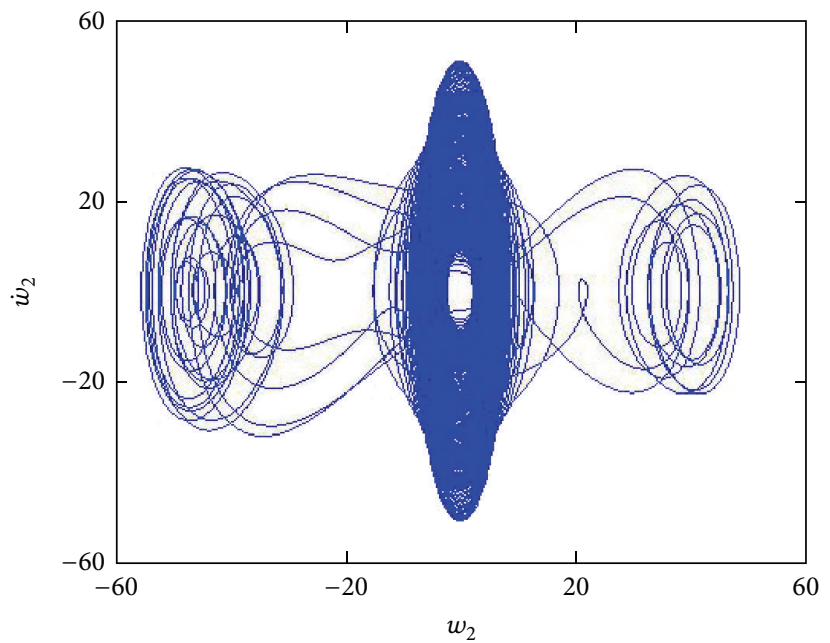

(c)

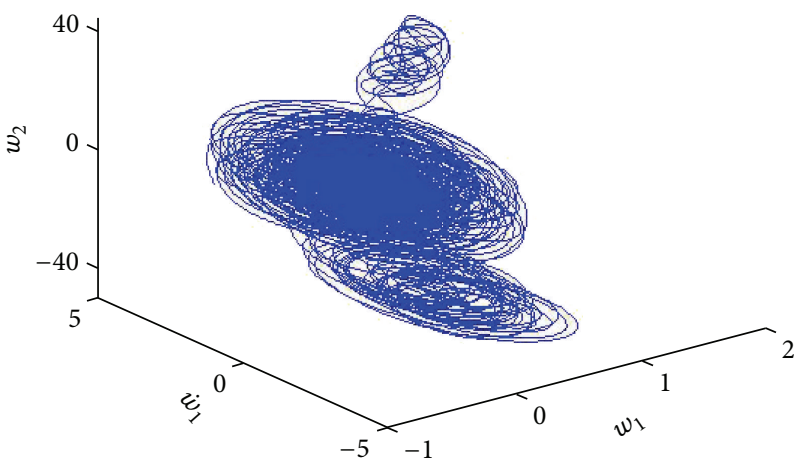

(e)

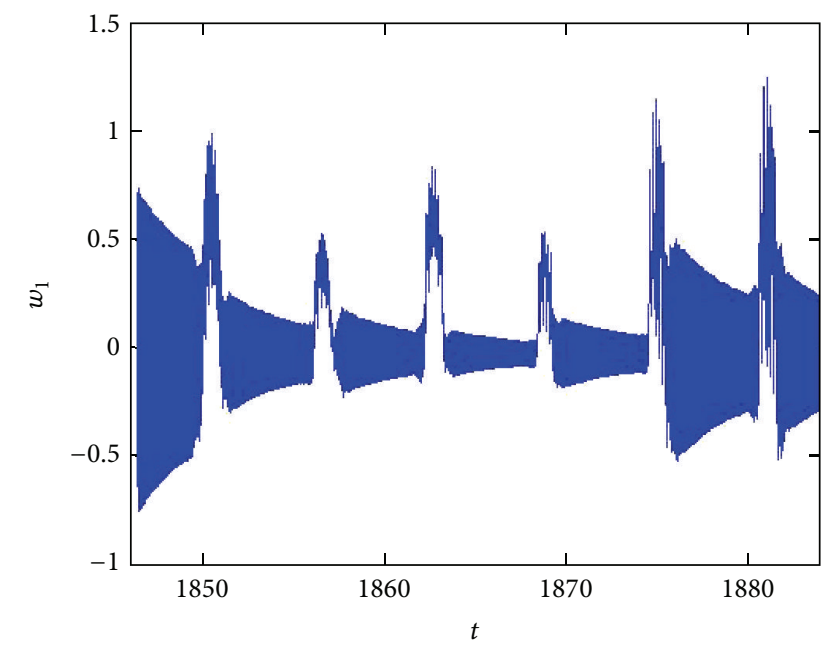

(b)

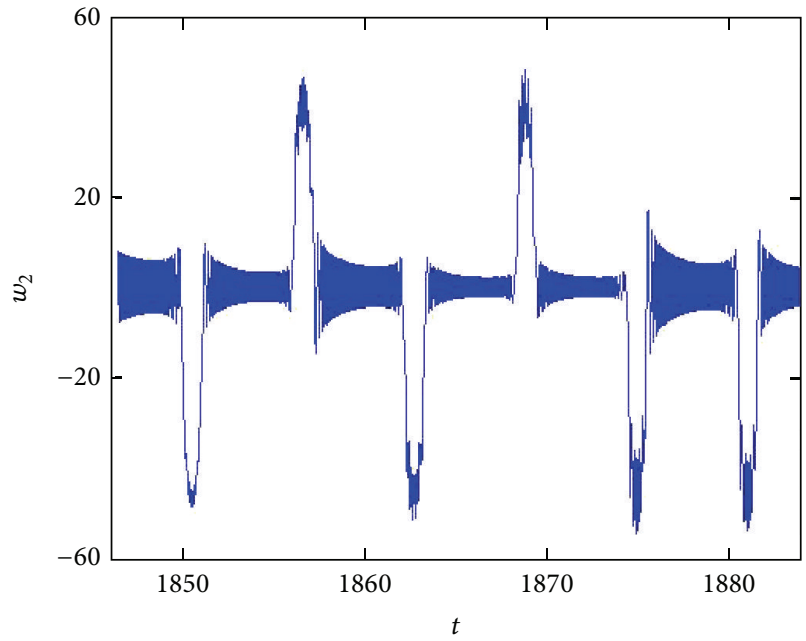

(d)

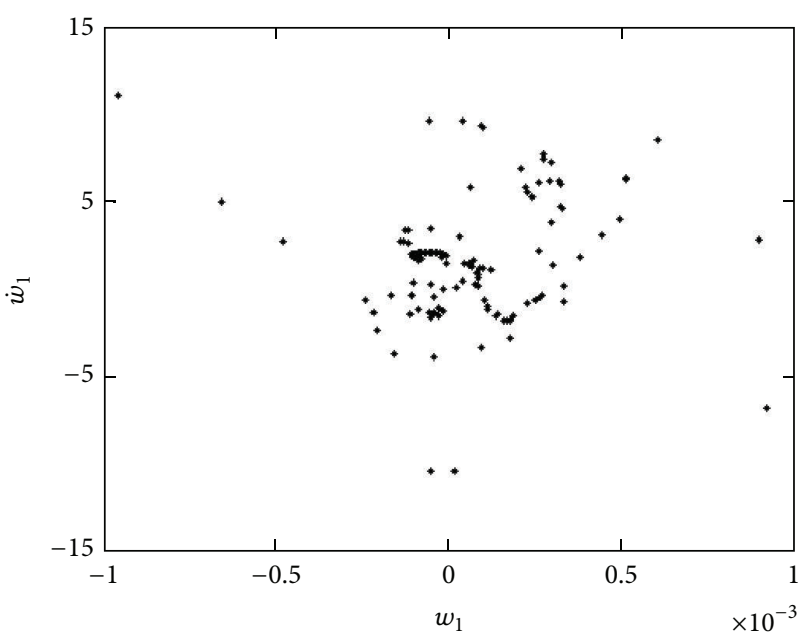

(f)

FIGURE 8: The chaotic motion of the FGM cylindrical panel occurs when the magnitude of the in-plane excitation is taken to be $7.79 \times 10^{7}$. The volume fraction index is taken to be 0.5 . The Mach number is taken to be 10.0 . 
be detected. Figure 8 indicates that the chaotic motion of the FGM cylindrical panel occurs.

\section{Conclusions}

The nonlinear dynamics of an FGM cylindrical panel under a hypersonic flow are presented. The material properties are graded continuously throughout the thickness of the panel according to the power law function and are temperature dependent. A third-order piston theory is applied for the hypersonic aerodynamic load. The Von Karman larger deflection theory in conjunction with energy approach is used to obtain the equations of motion. The bifurcation diagrams, phase portraits, time responses, and the Poincare section are employed to understand the periodic and chaotic motions of the cylindrical panel. It is obtained that, with the change of in-plane load parameter, the different nonlinear dynamics of the FGM panel occur. Different nonlinear dynamic behaviors alternate from stable periodic motion to instable chaotic motions in hypersonic flow. In addition, from the phase portraits and time responses, it can be seen that when the chaotic motion occurs, their phase portraits resemble each other in appearance for different Mach.

\section{Conflict of Interests}

The authors declare that there is no conflict of interests regarding the publication of this paper.

\section{Acknowledgments}

The authors acknowledge the financial support of the National Natural Science Foundation of China through Grants nos. 11272063, 11102226, and 11472298, the Science Foundation of Beijing Municipal Education Commission through Grant no. cit\&tcd201304112, and Foundation of Tianjin City through Grant no. 13JCQNJC04400.

\section{References}

[1] E. Efraim and M. Eisenberger, "Exact vibration analysis of variable thickness thick annular isotropic and FGM plates," Journal of Sound and Vibration, vol. 299, no. 4-5, pp. 720-738, 2007.

[2] R. A. Arciniega and J. N. Reddy, "Large deformation analysis of functionally graded shells," International Journal of Solids and Structures, vol. 44, no. 6, pp. 2036-2052, 2007.

[3] X. Guo and C. Mei, "Application of aeroelastic modes on nonlinear supersonic panel flutter at elevated temperatures," Computers and Structures, vol. 84, no. 24-25, pp. 1619-1628, 2006.

[4] L. K. Abbas, X. Rui, P. Marzocca, M. Abdalla, and R. de Breuker, "A parametric study on supersonic/hypersonic flutter behavior of aero-thermo-elastic geometrically imperfect curved skin panel," Acta Mechanica, vol. 222, no. 1-2, pp. 41-57, 2011.

[5] T. Prakash and M. Ganapathi, "Supersonic flutter characteristics of functionally graded flat panels including thermal effects," Composite Structures, vol. 72, no. 1, pp. 10-18, 2006.
[6] K.-J. Sohn and J.-H. Kim, "Structural stability of functionally graded panels subjected to aero-thermal loads," Composite Structures, vol. 82, no. 3, pp. 317-325, 2008.

[7] H. H. Ibrahim, M. Tawfik, and M. Al-Ajmi, "Non-linear panel flutter for temperature-dependent functionally graded material panels," Computational Mechanics, vol. 41, no. 2, pp. 325-334, 2008.

[8] H. H. Ibrahim, M. Tawfik, and M. Al-Ajmi, "Thermal buckling and nonlinear flutter behavior of functionally graded material panels," Journal of Aircraft, vol. 44, no. 5, pp. 1610-1618, 2007.

[9] T. Prakash, M. K. Singha, and M. Ganapathi, "A finite element study on the large amplitude flexural vibration characteristics of FGM plates under aerodynamic load," International Journal of Non-Linear Mechanics, vol. 47, no. 5, pp. 439-447, 2012.

[10] M. Hosseini and S. A. Fazelzadeh, "Aerothermoelastic postcritical and vibration analysis of temperature-dependent functionally graded panels," Journal of Thermal Stresses, vol. 33, no. 12, pp. 1188-1212, 2010.

[11] S. L. Lee and J. H. Kim, "Thermal post-buckling and limitcycle oscillation of functionally graded panel with structural damping in supersonic airflow," Composite Structures, vol. 91, no. 2, pp. 205-211, 2009.

[12] C. Mey, K. Abdel-Motagaly, and R. Chen, "Review of nonlinear panel flutter at supersonic and hypersonic speeds," Applied Mechanics Reviews, vol. 52, no. 10, pp. 321-332, 1999.

[13] M. Amabili, Nonlinear Vibrations and Stability of Shells and Plates, Cambridge University Press, New York, NY, USA, 2008.

[14] P. Marzocca, S. A. Fazelzadeh, and M. Hosseini, "A review of nonlinear aero-thermo-elasticity of functionally graded panels," Journal of Thermal Stresses, vol. 34, no. 5-6, pp. 536-568, 2011.

[15] M. Hosseini, S. A. Fazelzadeh, and P. Marzocca, "Chaotic and bifurcation dynamic behavior of functionally graded curved panels under aero-thermal loads," International Journal of Bifurcation and Chaos, vol. 21, no. 3, pp. 931-954, 2011.

[16] L. Librescu, P. Marzocca, and W. A. Silva, "Supersonic/hypersonic flutter and postflutter of geometrically imperfect circular cylindrical panels," Journal of Spacecraft and Rockets, vol. 39, no. 5, pp. 802-812, 2002.

[17] S. Mahmoudkhani, H. Haddadpour, and H. M. Navazi, "Supersonic flutter prediction of functionally graded conical shells," Composite Structures, vol. 92, no. 2, pp. 377-386, 2010.

[18] P. P. Friedmann, J. J. McNamara, B. J. Thuruthimattam, and I. Nydick, "Aeroelastic analysis of hypersonic vehicles," Journal of Fluids and Structures, vol. 19, no. 5, pp. 681-712, 2004.

[19] Y. S. Touloukian, Thermophysical Properties of High Temperature Solid Materials, Mac Millian, New York, NY, USA, 1967.

[20] W. Zhang, Y. X. Hao, and J. Yang, "Nonlinear dynamics of FGM circular cylindrical shell with clamped-clamped edges," Composite Structures, vol. 94, no. 3, pp. 1075-1086, 2012.

[21] C. Mei, K. Abdel-Moltagaly, and R. Chen, "Review of nonlinear panel flutter at supersonic and hypersonic speeds," Applied Mechanics Reviews, vol. 52, no. 10, pp. 321-332, 1999.

[22] M. J. Lighthill, "Oscillating airfoils at high Mach number," Journal of the Aeronautical Sciences, vol. 20, no. 6, pp. 402-406, 1953.

[23] H. Ashley and G. Zartarian, "Piston theory-a new aerodynamic tool for the aeroelastician," vol. 23, pp. 1109-1118, 1956.

[24] D. Cao and N. Zhao, "Active control of supersonic/hypersonic aeroelastic flutter for a two-dimensional airfoil with flap," Science China Technological Sciences, vol. 54, no. 8, pp. 19431953, 2011. 
[25] J. N. Reddy, Mechanics of Laminated Composite Plates and Shells: Theory and Analysis, CRC Press, 2004.

[26] R. Kadoli and N. Ganesan, "Buckling and free vibration analysis of functionally graded cylindrical shells subjected to a temperature-specified boundary condition," Journal of Sound and Vibration, vol. 289, no. 3, pp. 450-480, 2006.

[27] A. Bhimaraddi, "Large amplitude vibrations of imperfect antisymmetric angle-ply laminated plates," Journal of Sound and Vibration, vol. 162, no. 3, pp. 457-470, 1993.

[28] A. Nosier and J. N. Reddy, "A study of non-linear dynamic equations of higher-order shear deformation plate theories," International Journal of Non-Linear Mechanics, vol. 26, no. 2, pp. 233-249, 1991.

[29] J. N. Reddy, "Analysis of functionally graded plates," International Journal for Numerical Methods in Engineering, vol. 47, no. 1-3, pp. 663-684, 2000.

[30] H.-S. Shen, "Nonlinear bending response of functionally graded plates subjected to transverse loads and in thermal environments," International Journal of Mechanical Sciences, vol. 44, no. 3, pp. 561-584, 2002.

[31] L. Librescu, P. Marzocca, and W. A. Silva, "Linear/nonlinear supersonic panel flutter in a high-temperature field," Journal of Aircraft, vol. 41, no. 4, pp. 918-924, 2004.

[32] Y. X. Hao, W. Zhang, J. Yang, and S. Y. Li, "Nonlinear dynamic response of a simply supported rectangular functionally graded material plate under the time-dependent thermalmechanical loads," Journal of Mechanical Science and Technology, vol. 25, no. 7, pp. 1637-1646, 2011.

[33] Y. X. Hao, W. Zhang, and J. Yang, "Periodic and chaotic motions of FGM thin plate with two simply supported opposite and two free edges," International Journal of Bifurcation and Chaos, vol. 21, no. 6, pp. 1737-1753, 2011. 

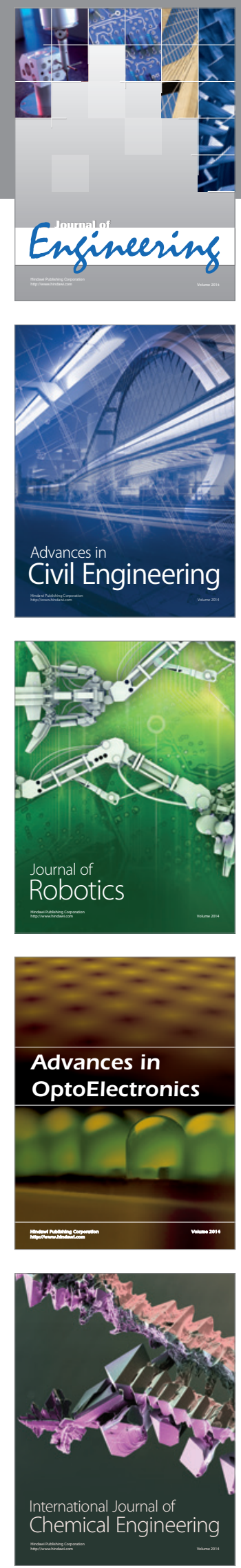

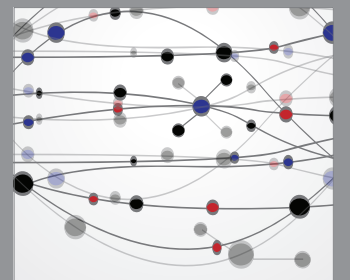

The Scientific World Journal
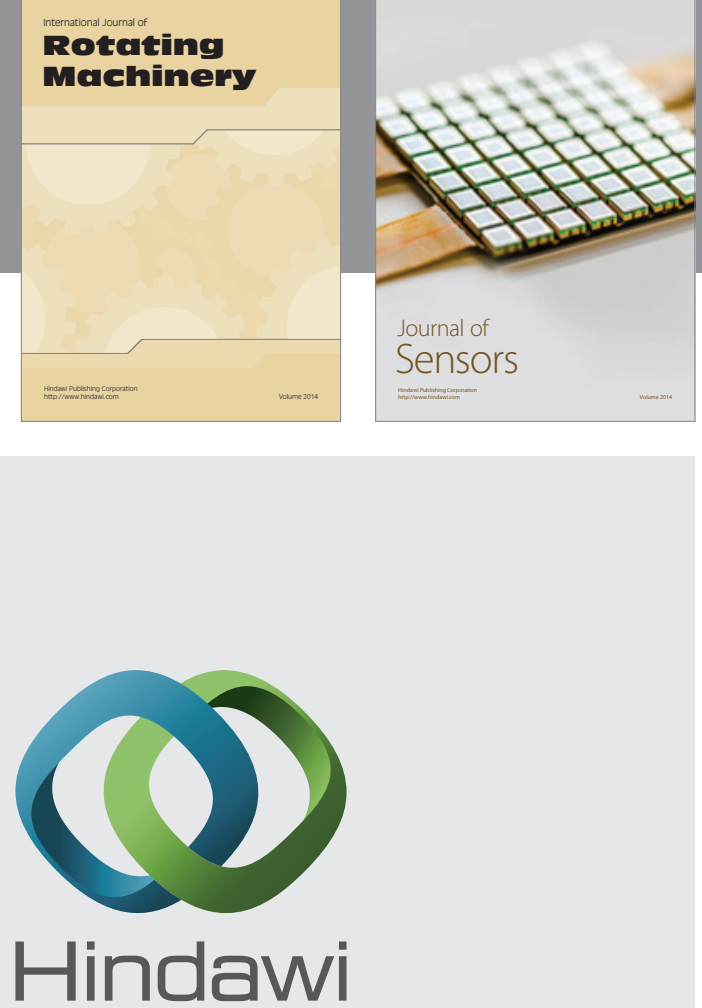

Submit your manuscripts at http://www.hindawi.com
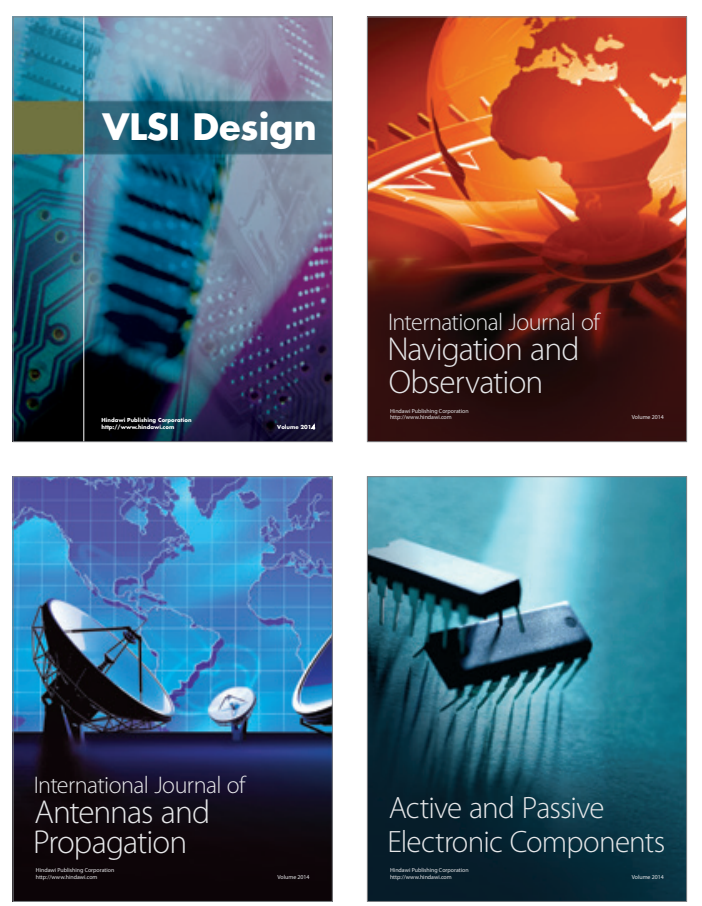
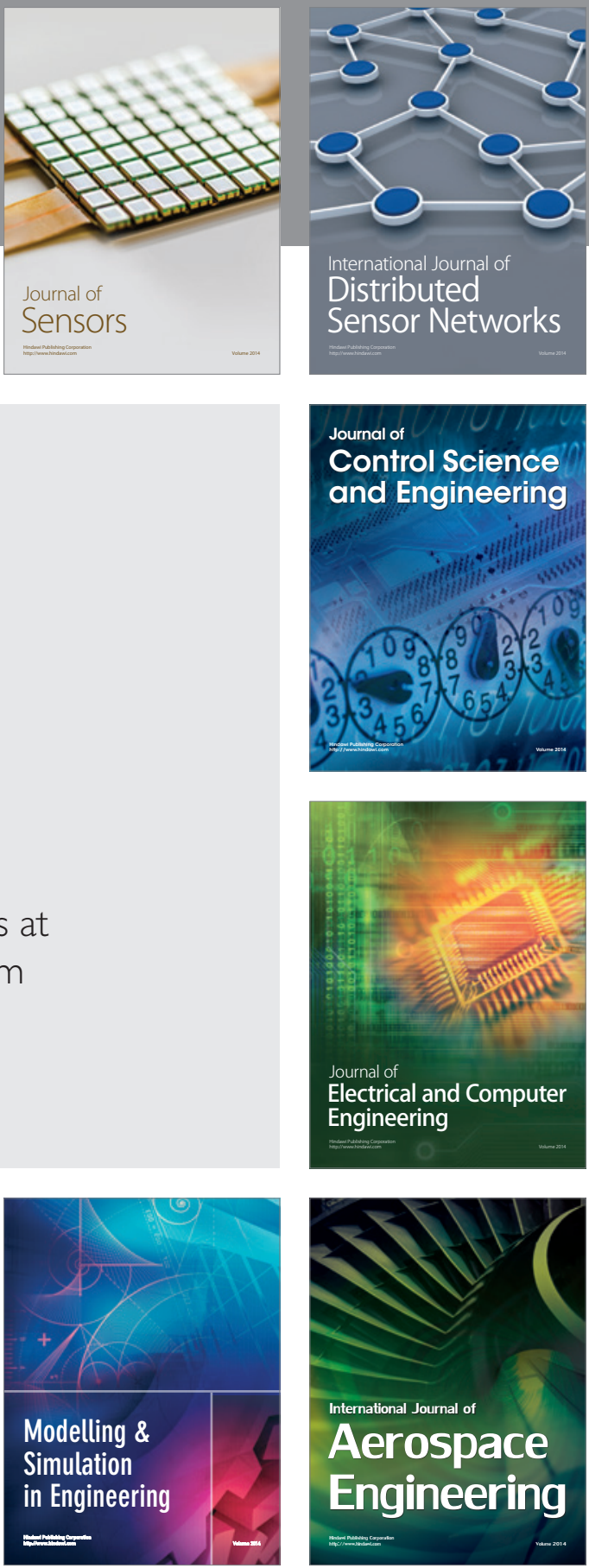

Journal of

Control Science

and Engineering
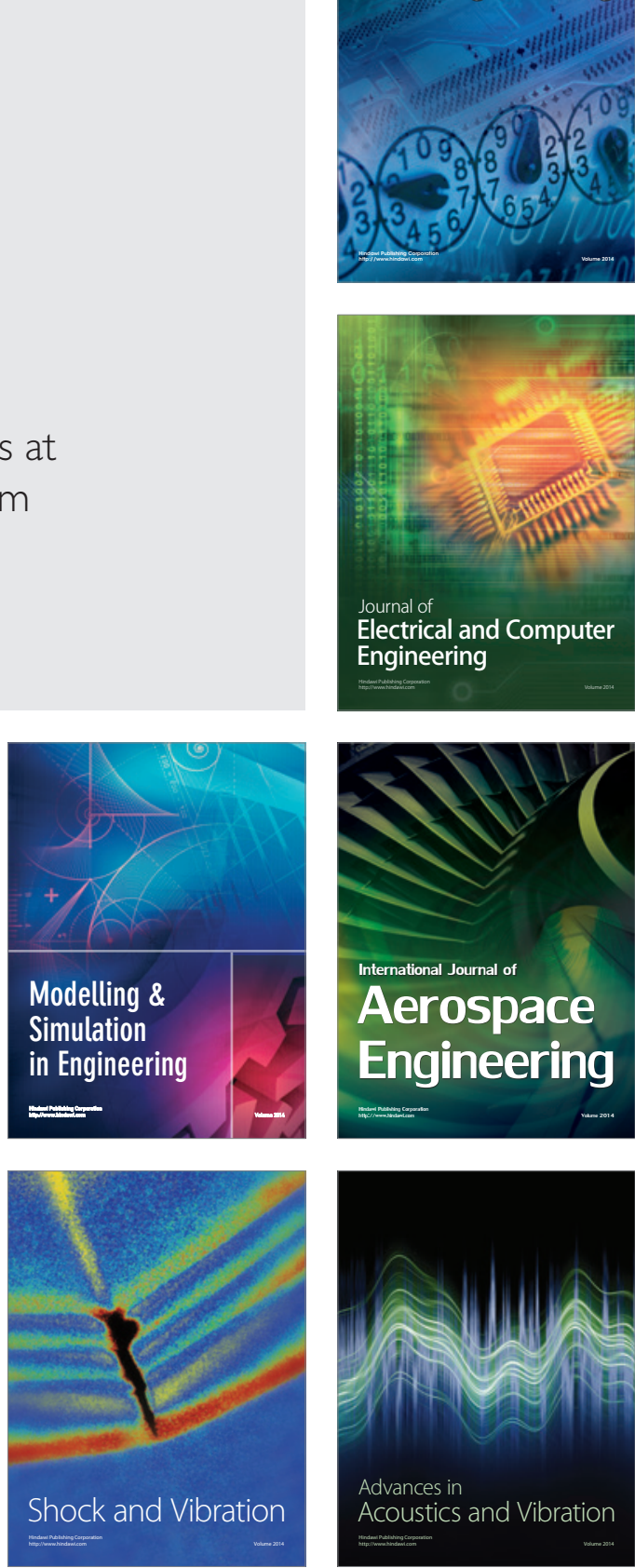